\title{
R-matrix electron-impact excitation data for the O-like iso-electronic sequence
}

\author{
Junjie Mao' ${ }^{1}$, N. R. Badnell ${ }^{1}$, and G. Del Zanna ${ }^{2}$ \\ ${ }^{1}$ Department of Physics, University of Strathclyde, Glasgow G4 0NG, UK \\ e-mail: junjie.mao@strath.ac.uk \\ 2 Department of Applied Mathematics and Theoretical Physics, University of Cambridge, Cambridge CB3 0WA, UK
}

Received 3 June 2021 / Accepted 14 July 2021

\begin{abstract}
Context. Astrophysical plasma codes are built on atomic databases. In the current atomic databases, R-matrix electron-impact excitation data of O-like ions are limited. The accuracy of plasma diagnostics with O-like ions depends on the availability and accuracy of the atomic data. This is particularly relevant in the context of future observatories equipped with the next generation of high-resolution spectrometers.

Aims. We aim to obtain level-resolved effective collision strengths of O-like ions from Ne III to $\mathrm{Zn} \mathrm{XXIII} \mathrm{(i.e.} \mathrm{Ne}^{2+}$ to $\mathrm{Zn}^{22+}$ ) over a wide range of temperatures. This includes transitions up to $n l=5 d$ for each ion. We also aim to assess the accuracy of the new data, as well as their impact on solar atmosphere plasma diagnostics, compared to those available within the CHIANTI database.

Methods. Large-scale R-matrix intermediate coupling frame transformation calculations were performed systematically for the O-like iso-electronic sequence. For each ion, 630 fine-structure levels were included in both the configuration interaction target and closecoupling collision expansions.

Results. Our results (energy levels, oscillator strengths, and effective collision strengths) of selected ions across the iso-electronic sequence are compared with those in archival databases and the literature. For the selected ions throughout the iso-electronic sequence, we find general agreement with the few previous R-matrix calculations of collision strengths. We illustrate the improvements for a few solar plasma diagnostics over existing CHIANTI atomic models based on distorted wave (DW) data. The electron-impact excitation data are archived according to the Atomic Data and Analysis Structure (ADAS) data class adf04 and will be available in OPEN-ADAS.
\end{abstract}

Key words. atomic data - techniques: spectroscopic - Sun: corona

\section{Introduction}

Spectral lines of O-like ions can be used to constrain the physical properties (e.g. temperature, density, and elemental abundance) of astrophysical plasmas. For instance, in the active region of the solar corona, the Fe XIX $\lambda 1118$ emission line is observed to study the hot $\left(10^{6} \mathrm{~K}\right)$ plasma emission (Feldman et al. 1973; Wang et al. 2006; Del Zanna et al. 2021b). The Fe XIX $13.42 \AA$, $13.52 \AA$, and $13.74 \AA$ emission lines from the ground and metastable levels are also observed in the Sun (Phillips et al. 1982). In the hot corona of the spectroscopic binary Procyon, the observed ground and metastable emission lines of S IX $47.25 \AA$, $55.54 \AA$, and $56.33 \AA$ were used for density diagnostics (Li et al. 2013). In the high-mass X-ray binary Vera X-1, Mg V $9.81 \AA$ was detected in absorption, while Si VII $7.06 \AA$ and S IX $5.32 \AA$ was detected in emission among other lines of $\mathrm{Ne}, \mathrm{Mg}$, and $\mathrm{Si}$ (Grinberg et al. 2017; Amato et al. 2021). These lines reveal a highly variable structured accretion flow close to the compact object (Grinberg et al. 2017), as well as a multi-phase wind of the companion star (Amato et al. 2021).

From a theoretical perspective, Raju \& Dwivedi (1978) studied the density dependence of solar emission lines of O-like ions (Ne III, Mg V, Si VII, S IX, Ar XI). Mao et al. (2017) presented density diagnostics with the ground and metastable absorption lines of Be-like to $\mathrm{C}$-like ions in the context of ionized winds driven away from active galactic nuclei. Because key diagnostic transitions (in emission and absorption) are in general weak
(Del Zanna \& Mason 2018; Mao et al. 2017), future observatories equipped with the next generation of high-resolution spectrometers (Smith et al. 2016; Barret et al. 2018; Cui et al. 2020) are certainly required to observe these lines.

Astrophysical plasma codes built on extensive atomic databases (e.g. $\mathrm{ADAS}^{1}, \mathrm{AtomDB}^{2}, \mathrm{CHIANTI}^{3}$, and $\mathrm{SPEX}^{4}$ ) enable plasma diagnostics with high-quality spectra obtained with current and future generations of high-resolution spectrometers. Continuous development of the atomic databases is essential because the current databases are neither as complete nor as accurate as we would wish.

Electron-impact excitation is one of the fundamental atomic processes to determine the level population of an ion. Systematic R-matrix intermediate coupling frame transformation (ICFT) calculations of the electron-impact excitation data have been performed for many iso-electronic sequences (Li-, Be-, B-, F-, $\mathrm{Ne}-, \mathrm{Na}-$, and Mg-like) since 2007 (see Badnell et al. 2016, for a review). Data for $\mathrm{C}$-like and $\mathrm{N}$-like iso-electronic sequences were recently presented in Mao et al. (2020a) and Mao et al. (2020b). The present work focuses on the O-like iso-electronic sequence. R-matrix electron-impact excitation data are available only for a few O-like ions. The number of energy levels (thus

\footnotetext{
1 http://www.adas.ac.uk

2 http://www . atomdb.org/Webguide/webguide.php

3 https://www. chiantidatabase.org/

4 https://www. sron.nl/astrophysics-spex
} 
the transitions among the levels) and temperature range of the effective collision strength vary among existing calculations.

Here we present systematic R-matrix calculations for O-like ions from $\mathrm{Ne}$ III to $\mathrm{Zn}$ XXIII (i.e. $\mathrm{Ne}^{2+}$ to $\mathrm{Zn}^{22+}$ ). We obtain effective collision strengths at 630 levels over a temperature range spanning five orders of magnitude for each ion. We describe the R-matrix calculation in Sect. 2. The results are summarized in Sect. 3. We compare the new data with results of previous works in Sect. 4. We also show the impact of new data on solar atmosphere plasma diagnostics. A summary is provided in Sect. 5. Additionally, a supplementary package is available at Zenodo (Mao 2021), which includes the input files of the R-matrix calculations, atomic data from the present work, archival databases, and literature, as well as the scripts we used to create the figures presented in this paper.

\section{Method}

We used the same method for the structure and collision calculations as described in Mao et al. (2020a) and Mao et al. (2020b) for C- and N-like ions. The main difference is that, for O-like ions, we included a total of 630 fine-structure levels in both the configuration-interaction target expansion and the close-coupling collision expansion. These levels arise from the 27 configurations listed in Table 1.

\subsection{Structure}

The AUTOSTRUCTURE code (Badnell 2011) was used to calculate the target atomic structure. By diagonalizing the BreitPauli Hamiltonian (Eissner et al. 1974), wave functions were calculated. The one-body relativistic terms (mass-velocity, nuclear plus Blume \& Watson spin-orbit, and Darwin) were included perturbatively. The Thomas-Fermi-Dirac-Amaldi model was used for the electronic potential with $n l$-dependent scaling parameters (Nussbaumer \& Storey 1978), as shown in Table A.1. These scaling parameters were obtained in the same way for all the ions in the iso-electronic sequence without further manual adjustment.

As recognized in Mao et al. (2020a) and Mao et al. (2020b), our calculation leads to a relatively poor structure for low-charge ions (e.g., Ne III and Mg V) and low-lying energy levels. This is limited by the use of the unique set of non-relativistic orthogonal orbitals (Berrington et al. 1995), which is required by the ICFT R-matrix scattering calculation.

\subsection{Collision}

For the ICFT R-matrix collision calculation, angular momenta up to $2 J=23$ and $2 J=77$ were included for the exchange and non-exchange calculations, respectively. Higher angular momenta (up to infinity) were included following the top-up formula of the Burgess sum rule (Burgess 1974) for dipole-allowed transitions and a geometric series for the non-dipole-allowed transitions (Badnell \& Griffin 2001).

Three sets of outer-region R-matrix calculations were used for each ion. First, the resonance region was sampled with a fine energy mesh. The sampling points increased with increasing atomic number, ranging from $\sim 3600$ for Ne III to $\sim 30000$ for Zn XXIII. Second, the energy range between the last threshold and three times the ionization potential was sampled with $\sim 1000$ points for all the ions in the iso-electronic sequence. Third, a non-exchange calculation between the first threshold and three times the ionization potential was performed, with
Table 1. List of configurations used for the structure and collision calculations.

\begin{tabular}{clclcl}
\hline \hline Index & Conf. & Index & Conf. & Index & Conf. \\
\hline 1 & $2 s^{2} 2 p^{4}$ & 2 & $2 s 2 p^{5}$ & 3 & $2 p^{6}$ \\
4 & $2 s^{2} 2 p^{3} 3 s$ & 5 & $2 s^{2} 2 p^{3} 3 p$ & 6 & $2 s^{2} 2 p^{3} 3 d$ \\
7 & $2 s 2 p^{4} 3 s$ & 8 & $2 s 2 p^{4} 3 p$ & 9 & $2 s 2 p^{4} 3 d$ \\
10 & $2 p^{5} 3 s$ & 11 & $2 p^{5} 3 p$ & 12 & $2 p^{5} 3 d$ \\
13 & $2 s^{2} 2 p^{3} 4 s$ & 14 & $2 s^{2} 2 p^{3} 4 p$ & 15 & $2 s^{2} 2 p^{3} 4 d$ \\
16 & $2 s^{2} 2 p^{3} 4 f$ & 17 & $2 s 2 p^{4} 4 s$ & 18 & $2 s^{4} p^{4} 4 p$ \\
19 & $2 s^{2} p^{4} 4 d$ & 20 & $2 s 2 p^{4} 4 f$ & 21 & $2 p^{5} 4 s$ \\
22 & $2 p^{5} 4 p$ & 23 & $2 p^{5} 4 d$ & 24 & $2 p^{5} 4 f$ \\
25 & $2 s^{2} 2 p^{3} 5 s$ & 26 & $2 s^{2} 2 p^{3} 5 p$ & 27 & $2 s^{2} 2 p^{3} 5 d$ \\
\hline
\end{tabular}

$\sim 1400$ sampling points for all ions in the iso-electronic sequence. Unresolved resonances in the ordinary collision strength in the resonance region were removed for the non-exchange calculation.

By convolving the ordinary collision strength $\left(\Omega_{i j}\right)$ with the Maxwellian energy distribution, we obtain the effective collision strength $\left(\Upsilon_{i j}\right)$ :

$\Upsilon_{i j}=\int \Omega_{i j} \exp \left(-\frac{E}{k T}\right) \mathrm{d}\left(\frac{E}{k T}\right)$

where $E$ is the kinetic energy of the scattered free electron, $k$ is the Boltzmann constant, and $T$ is the electron temperature of the plasma. To complete the Maxwellian convolution (Eq. (1)) at high temperatures, we calculated the infinite-energy Born and dipole line strength limits using AUTOSTRUCTURE. Between the last calculated energy point and the two limits, interpolation was used according to the type of transition in the BurgessTully scaled domain (i.e., the quadrature of the reduced collision strength over reduced energy; see Burgess \& Tully 1992).

\section{Results}

We have obtained R-matrix electron-impact excitation data for the O-like iso-electronic sequence from $\mathrm{Ne}$ III to $\mathrm{Zn}$ XXIII (i.e., $\mathrm{Ne}^{2+}$ and $\mathrm{Zn}^{22+}$ ). Our effective collision strengths cover five orders of magnitude in temperature $(z+1)^{2}\left(2 \times 10^{1}, 2 \times 10^{6}\right) \mathrm{K}$, where $z$ is the ionic charge (e.g., $z=7$ for Si VIII).

The effective collision strength data will be archived according to the Atomic Data and Analysis Structure (ADAS) data class $a d f 04$ and will be available in OPEN-ADAS and our UKAPAP website ${ }^{5}$. These data can be used to improve the atomic database of astrophysical plasma codes, such as CHIANTI (Dere et al. 1997; Del Zanna et al. 2021a) and SPEX (Kaastra et al. 1996, 2020), where no data or less accurate data were available. The ordinary collision strength data will also be archived in OPEN-ADAS ${ }^{6}$.

\section{Discussion}

Several ions across the iso-electronic sequence were selected to assess the quality of our structure and collision calculations. These ions were selected because detailed results from

\footnotetext{
5 http://apap-network.org/

6 http://open.adas.ac.uk/
} 

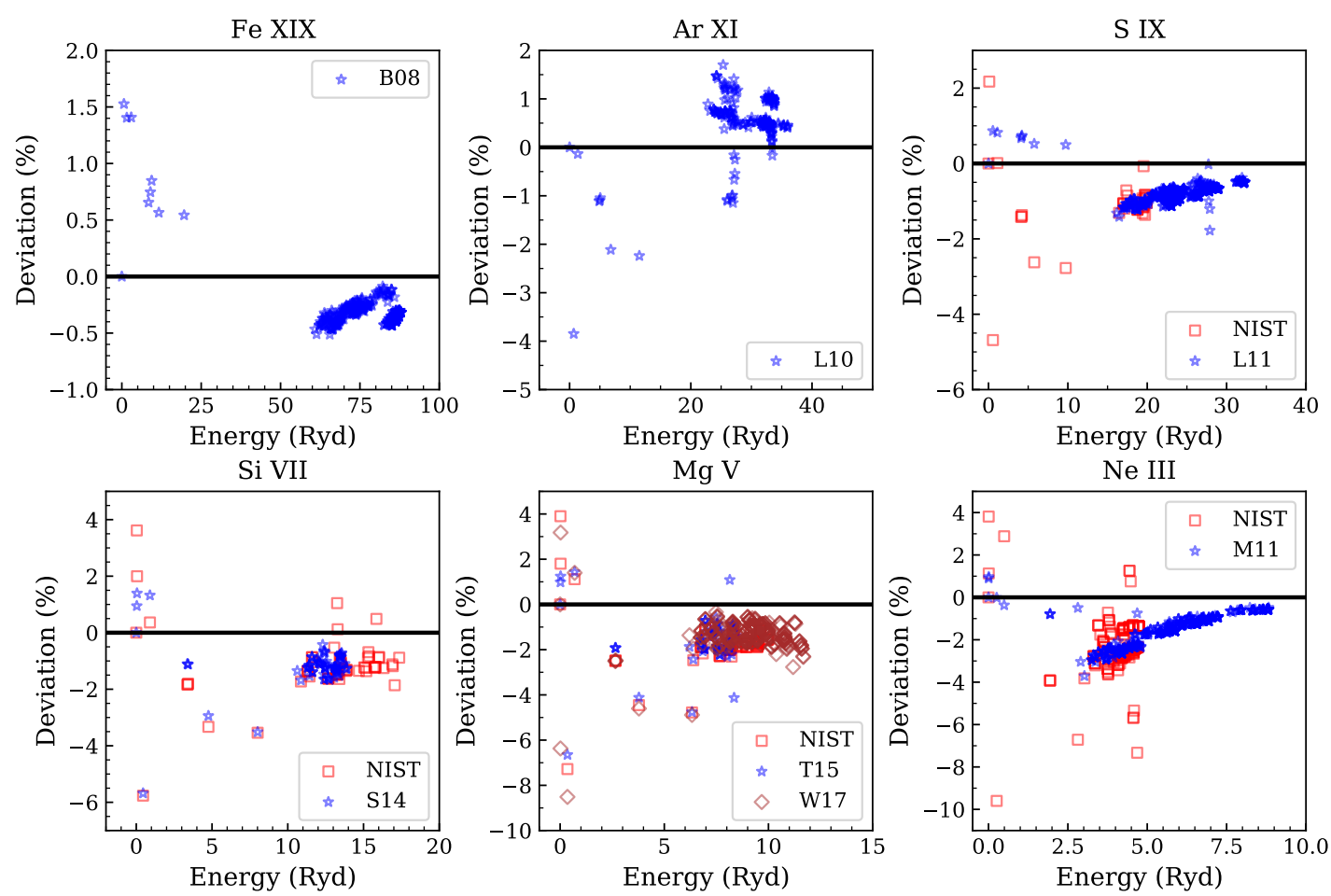

Fig. 1. Percentage deviations between the energy levels used here (horizontal lines in black), the experimental levels (NIST), and previous works: B08 refers to Butler \& Badnell (2008), L10 refers to Ludlow et al. (2010), L11 refers to Liang et al. (2011), S14 refers to Sossah \& Tayal (2014), T15 refers to Tayal \& Sossah (2015), W17 refers to Wang et al. (2017), and M11 refers to McLaughlin et al. (2011).

archival databases (NIST ${ }^{7}$ and OPEN-ADAS) and the literature are available for comparison purposes.

First, we compare the energy levels among NIST, previous works, and our work. As shown in Fig. 1, generally speaking, the energy levels agree within $\sim 5 \%$ for the high-charge ions (e.g. Fe XIX and Ar XI). A larger deviation $(\lesssim 10 \%)$ is found for lowcharge ions such as Ne III, in particular, for some of the low-lying energy levels.

Second, we compare transition strengths $\log (g f)$, where $g$ and $f$ are the statistical weight and oscillator strength of the transition, respectively. Figure 2 shows the deviation of the transition strengths $\Delta \log (g f)$ in archival databases and previous works with respect to our work. We limit our comparison to relatively strong transitions with $g f \gtrsim 10^{-6}$ from the lowest five energy levels of the ground configuration: $2 \mathrm{~s}^{2} 2 \mathrm{p}^{4} \quad\left({ }^{3} \mathrm{P}_{2,1,0},{ }^{1} \mathrm{D}_{2},{ }^{1} \mathrm{~S}_{0}\right)$. For low-density astrophysical plasmas, the ground and first four metastable levels (Mao et al. 2017) dominate the level population. Weak transitions associated with higher metastable levels have little impact on astrophysical plasma diagnostics. Large differences $(\Delta \log (g f) \gtrsim 1.0)$ are noted for some transitions. Some are caused by level mixing, for instance for Ar XI, level $692 \mathrm{~s}^{2} 2 \mathrm{p}^{3} 3 \mathrm{~d}\left({ }^{1} \mathrm{D}_{2}\right)$ with $E=26.84$ Ryd and level $872 \mathrm{~s}^{2} 2 \mathrm{p}^{3} 3 \mathrm{~d}\left({ }^{1} \mathrm{D}_{2}\right)$ with $E=27.41$ Ryd are mixed in our calculation. Some are associated with the last few energy levels in previous calculations, for example levels 76-89 of the 92-level calculation by Sossah \& Tayal (2014) for Si VII and levels 76-86 of the 86-level calculation by Tayal \& Sossah (2015) for Mg V. When different size-scale structure calculations are compared, high-lying levels can be subject to the significant effect of a different CI expansion (Del Zanna et al. 2015; Fernández-Menchero et al. 2016).

\footnotetext{
7 https://www.nist.gov/pml/atomic-spectra-database
}

Hexbin plots (Carr et al. 1987) are used to compare a large number of effective collision strengths (figures in Appendix B). The statistics of the hexbin plot comparison are provided in Table 2. In general, different calculations agree better at higher temperatures. At low and intermediate temperatures, the additional resonances included in our work contribute to most of the deviations.

In the following, we compare effective collision strength data for Fe XIX (Sect. 4.1), Ca XIII (Sect. 4.2), Ar XI (Sect. 4.3), S IX (Sect. 4.4), Si VII (Sect. 4.5), Mg V (Sect. 4.6), and Ne III (Sect. 4.7). We focus on the selected prominent allowed and forbidden transitions from the ground and metastable levels listed in Table 3.

\subsection{FeXIX}

The most recent calculation of R-matrix electron-impact excitation data for Fe XIX ( $\mathrm{Fr} \mathrm{Fe}^{18+}$ ) was presented by Butler \& Badnell (2008, B08 hereafter). B08 used AUTOSTRUCTURE for the structure calculation, as did we. The top left panels of Fig. 1 and Fig. 2 show that the energy levels and transition strengths agree well between our work and B08.

Like B08, we used the R-matrix ICFT method for the scattering calculation. B08 included 342 fine-structure levels in the close-coupling expansions, which is fewer levels than in our work (630 levels). Figure B.1 shows the hexbin plot comparison of the effective collision strengths at $T=1.80 \times 10^{5} \mathrm{~K}$, $3.61 \times 10^{6} \mathrm{~K}$, and $7.22 \times 10^{7} \mathrm{~K}$. The effective collision strengths for the six selected dipole transitions from the ground $(13.52 \AA$ and $108.36 \AA)$ and metastable $(13.42 \AA, 13.74 \AA, 109.95 \AA$, and $119.98 \AA$ ) levels, as well as two forbidden transitions (592.24 $\AA$ and 1118.06 $\AA$ ), agree well between our work and B08 (Fig. 3). 

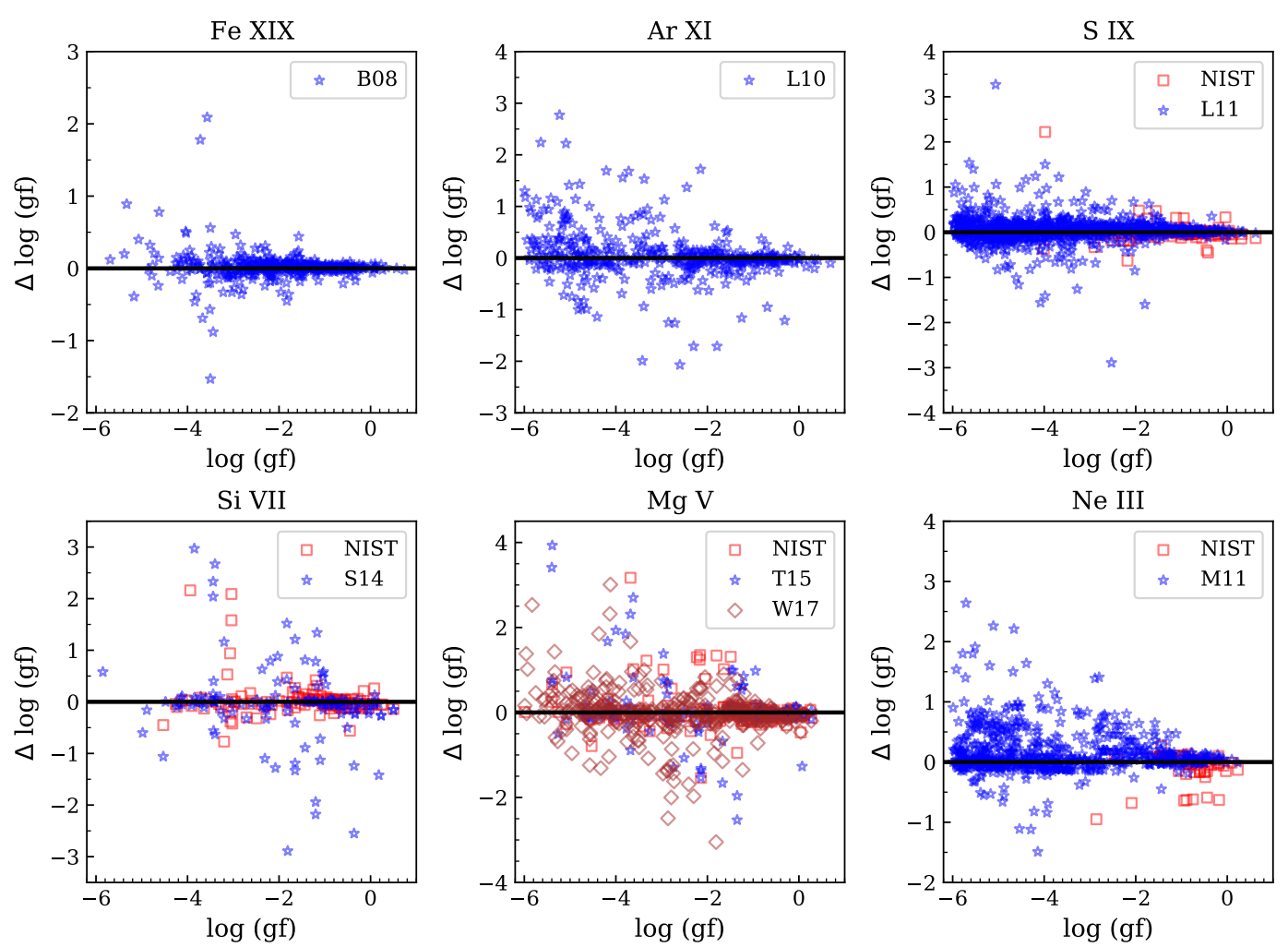

Fig. 2. Comparisons of $\log (g f)$ from our work (horizontal black line) with the experimental works (NIST) and previous works: B08 refers to Butler \& Badnell (2008), L10 refers to Ludlow et al. (2010), L11 refers to Liang et al. (2011), S14 refers to Sossah \& Tayal (2014), T15 refers to Tayal \& Sossah (2015), W17 refers to Wang et al. (2017), and M11 refers to McLaughlin et al. (2011). We note that this comparison is limited to relatively strong transitions with $\log (g f) \gtrsim-6$ originating from the lowest five energy levels.

Table 2. Statistics of the hexbin plot comparison of the effective collision strength comparison for Fe XIX (Butler \& Badnell 2008, B08), Ar XI (Ludlow et al. 2010, L10), S IX (Liang et al. 2011, L11), Si VII (Sossah \& Tayal 2014, S14), Mg V (Tayal \& Sossah 2015; Wang et al. 2017), and Ne III (McLaughlin et al. 2011, M11).

\begin{tabular}{ccccccc}
\hline \hline Ion & $T$ (low) & $T$ (middle) & $T$ (high) & $T$ (low) & $T$ (middle) & $T$ (high) \\
\hline Fe XIX (B08) & $\sim 5.3 \times 10^{4}(54 \%)$ & $\sim 4.9 \times 10^{4}(54 \%)$ & $\sim 4.3 \times 10^{4}(27 \%)$ & $1681(31 \%)$ & $1612(28 \%)$ & $1405(6 \%)$ \\
Ar XI (L10) & $\sim 2.4 \times 10^{4}(68 \%)$ & $\sim 2.3 \times 10^{4}(62 \%)$ & $\sim 2.1 \times 10^{4}(56 \%)$ & $1105(44 \%)$ & $1091(33 \%)$ & $1006(32 \%)$ \\
S IX (L11) & $4129(75 \%)$ & $4020(55 \%)$ & $3626(34 \%)$ & $444(29 \%)$ & $442(31 \%)$ & $441(23 \%)$ \\
Si VII (S14) & $4092(70 \%)$ & $4072(70 \%)$ & $4038(59 \%)$ & $443(35 \%)$ & $442(21 \%)$ & $407(20 \%)$ \\
Mg V (W17) & $\sim 4.7 \times 10^{4}(51 \%)$ & $\sim 4.7 \times 10^{4}(48 \%)$ & $\sim 4.6 \times 10^{4}(47 \%)$ & $1523(29 \%)$ & $1515(20 \%)$ & $1505(22 \%)$ \\
Mg V (T15) & $3560(62 \%)$ & $3547(61 \%)$ & $3522(54 \%)$ & $415(26 \%)$ & $415(20 \%)$ & $415(18 \%)$ \\
Ne III (M11) & $\sim 1.5 \times 10^{5}(83 \%)$ & $\sim 1.5 \times 10^{5}(35 \%)$ & $\sim 1.4 \times 10^{5}(25 \%)$ & $2435(56 \%)$ & $2600(11 \%)$ & $2409(4 \%)$ \\
\hline
\end{tabular}

Notes. Cols. 2-4 give the number of transitions with $\log (\Upsilon)>-5$ in both data sets and the percentage of transitions with deviations larger than 0.2 dex at three temperatures (ion-dependent) used for the hexbin plots. Columns 5-7 are the statistics when the transitions from the lowest five energy levels are limited (i.e. the ground and the first four metastable levels). For Mg V, statistics of the comparisons with Tayal \& Sossah (2015, T15) and Wang et al. (2017, W17) are shown.

\subsection{CaXIII}

The collision data of Ca XIII in the CHIANTI database (v10 Del Zanna et al. 2021a) originate from Landi \& Bhatia (2005), who presented a DW calculation of 86 levels. Figure 4 compares the effective collision strength of selected allowed and forbidden transitions listed in Table 3. The two calculations agree well for the allowed transitions, but they differ for the forbidden transitions. This is similar to the case of C-like Ar XIII discussed in Mao et al. (2020a). Our rates result in a significant increase in the predicted line intensities of the UV forbidden lines, which have been observed by SOHO/SUMER, for instance.

\section{3. $\operatorname{Ar} X I$}

Ludlow et al. (2010, L10 hereafter) presented the most recent Rmatrix calculations of electron-impact excitation data for Ar XI (or $\mathrm{Ar}^{10+}$ ). Like L10, we used AUTOSTRUCTURE to calculate the structure. The energy levels of the L10 calculations are within $\sim 2-4 \%$ of our work (Fig. 1). The transition strengths agree well between L10 and our work. 

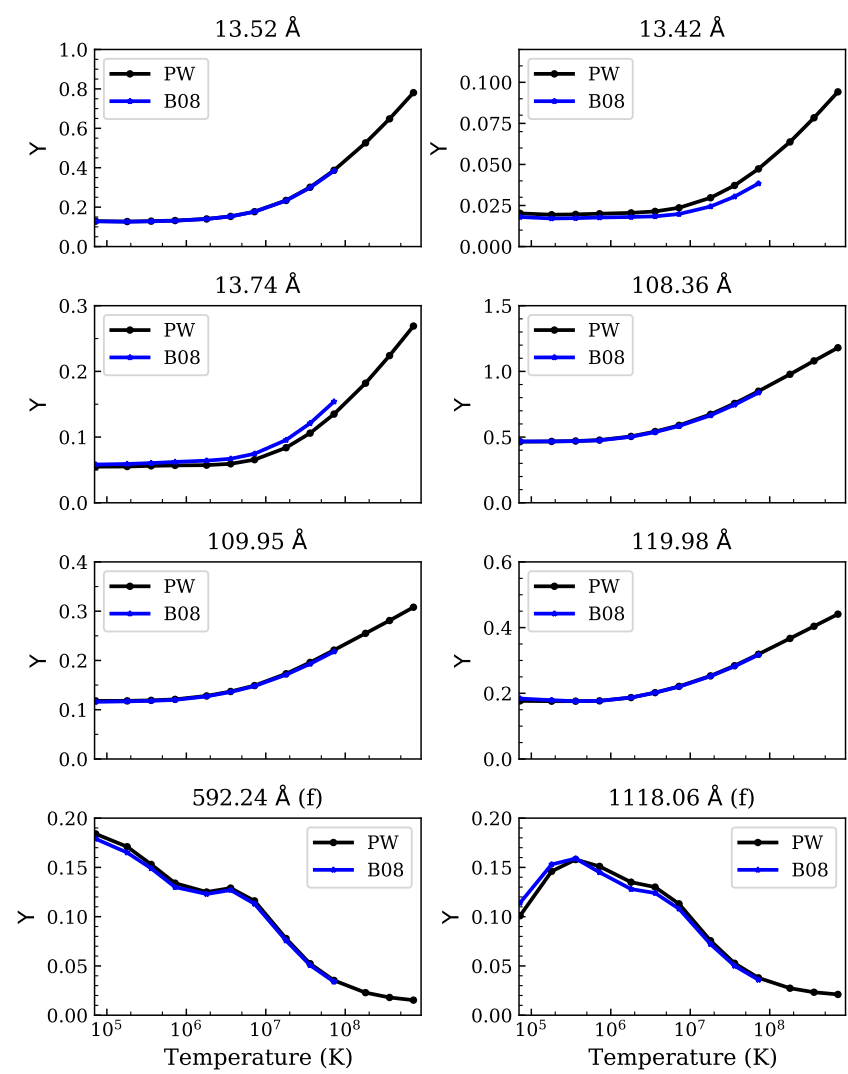

Fig. 3. Comparison of Fe XIX (or $\mathrm{Fe}^{18+}$ ) effective collision strengths between our work (PW) and Butler \& Badnell (2008, B08) for the selected dipole transitions from the ground (upper) and the metastable (middle and bottom) levels listed in Table 3.

$131.22 \AA$
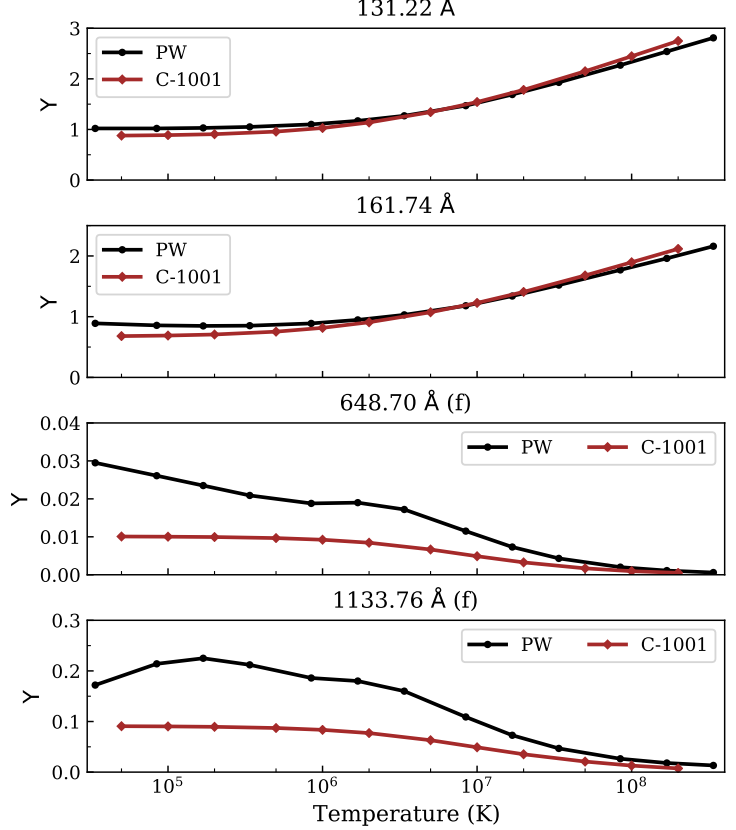

Fig. 4. Comparison of $\mathrm{Ca} X I I I$ (or $\mathrm{Ca}^{12+}$ ) effective collision strengths between our work (PW) and Landi \& Bhatia (2005, L05) as included in the CHIANTI atomic database v10.0.1 (C-1001). The top two panels are allowed transitions from the ground (131.22 $\AA$ ) and metastable $(161.74 \AA)$ levels. The bottom two panels are forbidden transitions (648.70 ̊ and $1133.76 \AA$ ).
Table 3. Selected prominent transitions from the lowest four energy levels for O-like ions.

\begin{tabular}{|c|c|c|c|}
\hline Ion & Lower level & Upper level & $\lambda_{0}(\AA)$ \\
\hline Fe XIX & $\begin{array}{l}2 \mathrm{~s}^{2} 2 \mathrm{p}^{4}\left({ }^{3} \mathrm{P}_{1}\right) \\
2 \mathrm{~s}^{2} 2 \mathrm{p}^{4}\left({ }^{3} \mathrm{P}_{2}\right) \\
2 \mathrm{~s}^{2} 2 \mathrm{p}^{4}\left({ }^{1} \mathrm{D}_{2}\right) \\
2 \mathrm{~s}^{2} 2 \mathrm{p}^{4}\left({ }^{3} \mathrm{P}_{2}\right) \\
2 \mathrm{~s}^{2} 2 \mathrm{p}^{4}\left({ }^{3} \mathrm{P}_{0}\right) \\
2 \mathrm{~s}^{2} 2 \mathrm{p}^{4}\left({ }^{3} \mathrm{P}_{1}\right) \\
2 \mathrm{~s}^{2} 2 \mathrm{p}^{4}\left({ }^{3} \mathrm{P}_{2}\right) \\
2 \mathrm{~s}^{2} 2 \mathrm{p}^{4}\left({ }^{3} \mathrm{P}_{2}\right)\end{array}$ & $\begin{array}{l}2 s^{2} 2 p^{3} 3 d\left({ }^{3} \mathrm{P}_{2}\right) \\
2 s^{2} 2 p^{3} 3 d\left({ }^{3} \mathrm{D}_{3}\right) \\
2 s^{2} 2 p^{3} 3 d\left({ }^{1} \mathrm{~F}_{3}\right) \\
2 s 2 p^{5}\left({ }^{3} \mathrm{P}_{2}\right) \\
2 \mathrm{~s}^{5} \mathrm{p}^{5}\left({ }^{3} \mathrm{P}_{1}\right) \\
2 \mathrm{~s} 2 \mathrm{p}^{5}\left({ }^{3} \mathrm{P}_{2}\right) \\
2 \mathrm{~s}^{2} 2 \mathrm{p}^{4}\left({ }^{1} \mathrm{D}_{2}\right) \\
2 \mathrm{~s}^{2} 2 \mathrm{p}^{4}\left({ }^{3} \mathrm{P}_{1}\right)\end{array}$ & $\begin{array}{l}13.42 \\
13.52(\mathrm{~g}) \\
13.74 \\
108.36(\mathrm{~g}) \\
109.95 \\
119.98 \\
592.24(\mathrm{~g}, \mathrm{f}) \\
1118.06(\mathrm{~g}, \mathrm{f})\end{array}$ \\
\hline Ca XIII & $\begin{array}{l}2 s^{2} 2 p^{4}\left({ }^{1} D_{2}\right) \\
2 s^{2} 2 p^{4}\left({ }^{3} P_{2}\right) \\
2 s^{2} 2 p^{4}\left({ }^{3} P_{1}\right) \\
2 s^{2} 2 p^{4}\left({ }^{3} P_{2}\right)\end{array}$ & $\begin{array}{l}2 \mathrm{~s} 2 \mathrm{p}^{5}\left({ }^{1} \mathrm{P}_{1}\right) \\
2 s 2 \mathrm{p}^{5}\left({ }^{3} \mathrm{P}_{2}\right) \\
2 \mathrm{~s}^{2} 2 \mathrm{p}^{4}\left({ }^{1} \mathrm{~S}_{0}\right) \\
2 \mathrm{~s}^{2} 2 \mathrm{p}^{4}\left({ }^{1} \mathrm{D}_{2}\right)\end{array}$ & $\begin{array}{l}131.22 \\
161.74(\mathrm{~g}) \\
648.70(\mathrm{f}) \\
1133.76(\mathrm{~g}, \mathrm{f})\end{array}$ \\
\hline Ar XI & $\begin{array}{l}2 s^{2} 2 p^{4}\left({ }^{1} D_{2}\right) \\
2 s^{2} 2 p^{4}\left({ }^{3} P_{2}\right) \\
2 s^{2} 2 p^{4}\left({ }^{3} P_{1}\right) \\
2 s^{2} 2 p^{4}\left({ }^{3} P_{2}\right)\end{array}$ & $\begin{array}{l}2 \mathrm{~s} 2 \mathrm{p}^{5}\left({ }^{1} \mathrm{P}_{1}\right) \\
2 \mathrm{~s} 2 \mathrm{p}^{5}\left({ }^{3} \mathrm{P}_{2}\right) \\
2 \mathrm{~s}^{2} 2 \mathrm{p}^{4}\left({ }^{1} \mathrm{~S}_{0}\right) \\
2 \mathrm{~s}^{2} 2 \mathrm{p}^{4}\left({ }^{1} \mathrm{D}_{2}\right)\end{array}$ & $\begin{array}{l}151.85 \\
188.81(\mathrm{~g}) \\
745.95(\mathrm{f}) \\
1392.10(\mathrm{~g}, \mathrm{f})\end{array}$ \\
\hline S IX & $\begin{array}{l}2 \mathrm{~s}^{2} 2 \mathrm{p}^{4}\left({ }^{3} \mathrm{P}_{0}\right) \\
2 \mathrm{~s}^{2} 2 \mathrm{p}^{4}\left({ }^{3} \mathrm{P}_{2}\right) \\
2 \mathrm{~s}^{2} 2 \mathrm{p}^{4}\left({ }^{1} \mathrm{D}_{2}\right) \\
2 \mathrm{~s}^{2} 2 \mathrm{p}^{4}\left({ }^{3} \mathrm{P}_{1}\right) \\
2 \mathrm{~s}^{2} 2 \mathrm{p}^{4}\left({ }^{1} \mathrm{D}_{2}\right) \\
2 \mathrm{~s}^{2} 2 \mathrm{p}^{4}\left({ }^{3} \mathrm{P}_{2}\right) \\
2 \mathrm{~s}^{2} 2 \mathrm{p}^{4}\left({ }^{3} \mathrm{P}_{1}\right) \\
2 \mathrm{~s}^{2} 2 \mathrm{p}^{4}\left({ }^{3} \mathrm{P}_{2}\right)\end{array}$ & $\begin{array}{l}2 s^{2} 2 p^{3} 3 d\left({ }^{3} \mathrm{D}_{1}\right) \\
2 s^{2} 2 p^{3} 3 d\left({ }^{3} \mathrm{P}_{2}\right) \\
2 s^{2} 2 p^{3} 3 s\left({ }^{1} \mathrm{D}_{2}\right) \\
2 s^{2} 2 p^{3} 3 s\left({ }^{3} \mathrm{~S}_{1}\right) \\
2 \mathrm{~s}^{5} \mathrm{p}^{5}\left({ }^{1} \mathrm{P}_{1}\right) \\
2 \mathrm{~s}^{2} \mathrm{p}^{5}\left({ }^{3} \mathrm{P}_{2}\right) \\
2 \mathrm{~s}^{2} 2 \mathrm{p}^{4}\left({ }^{1} \mathrm{~S}_{0}\right) \\
2 \mathrm{~s}^{2} 2 \mathrm{p}^{4}\left({ }^{1} \mathrm{D}_{2}\right)\end{array}$ & $\begin{array}{l}46.61 \\
47.25(\mathrm{~g}) \\
55.54 \\
56.33 \\
179.28 \\
224.73(\mathrm{~g}) \\
871.73(\mathrm{f}) \\
1715.41(\mathrm{~g}, \mathrm{f})\end{array}$ \\
\hline$\overline{\text { Si VII }}$ & $\begin{array}{l}2 s^{2} 2 p^{4}\left({ }^{1} D_{2}\right) \\
2 s^{2} 2 p^{4}\left({ }^{3} P_{2}\right) \\
2 s^{2} 2 p^{4}\left({ }^{3} P_{1}\right) \\
2 s^{2} 2 p^{4}\left({ }^{3} P_{2}\right)\end{array}$ & $\begin{array}{l}2 \mathrm{~s} 2 \mathrm{p}^{5}\left({ }^{1} \mathrm{P}_{1}\right) \\
2 \mathrm{~s} 2 \mathrm{p}^{5}\left({ }^{3} \mathrm{P}_{2}\right) \\
2 \mathrm{~s}^{2} 2 \mathrm{p}^{4}\left({ }^{1} \mathrm{~S}_{0}\right) \\
2 \mathrm{~s}^{2} 2 \mathrm{p}^{4}\left({ }^{1} \mathrm{D}_{2}\right)\end{array}$ & $\begin{array}{l}217.83 \\
275.36(\mathrm{~g}) \\
1049.15(\mathrm{f}) \\
2147.40(\mathrm{~g}, \mathrm{f})\end{array}$ \\
\hline $\mathrm{Mg} \mathrm{V}$ & $\begin{array}{l}2 s^{2} 2 p^{4}\left({ }^{1} D_{2}\right) \\
2 s^{2} 2 p^{4}\left({ }^{3} P_{2}\right) \\
2 s^{2} 2 p^{4}\left({ }^{3} P_{1}\right) \\
2 s^{2} 2 p^{4}\left({ }^{3} P_{2}\right)\end{array}$ & $\begin{array}{l}2 \mathrm{~s} 2 \mathrm{p}^{5}\left({ }^{1} \mathrm{P}_{1}\right) \\
2 \mathrm{~s} 2 \mathrm{p}^{5}\left({ }^{3} \mathrm{P}_{2}\right) \\
2 \mathrm{~s}^{2} 2 \mathrm{p}^{4}\left({ }^{1} \mathrm{~S}_{0}\right) \\
2 \mathrm{~s}^{2} 2 \mathrm{p}^{4}\left({ }^{1} \mathrm{D}_{2}\right)\end{array}$ & $\begin{array}{l}276.58 \\
353.09(\mathrm{~g}) \\
1324.43(\mathrm{f}) \\
2783.58(\mathrm{~g}, \mathrm{f})\end{array}$ \\
\hline $\mathrm{Ne}$ III & $\begin{array}{l}2 s^{2} 2 p^{4}\left({ }^{1} D_{2}\right) \\
2 s^{2} 2 p^{4}\left({ }^{3} P_{2}\right) \\
2 s^{2} 2 p^{4}\left({ }^{3} P_{1}\right) \\
2 s^{2} 2 p^{4}\left({ }^{3} P_{2}\right)\end{array}$ & $\begin{array}{l}2 \mathrm{~s} 2 \mathrm{p}^{5}\left({ }^{1} \mathrm{P}_{1}\right) \\
2 \mathrm{~s} 2 \mathrm{p}^{5}\left({ }^{3} \mathrm{P}_{2}\right) \\
2 \mathrm{~s}^{2} 2 \mathrm{p}^{4}\left({ }^{1} \mathrm{~S}_{0}\right) \\
2 \mathrm{~s}^{2} 2 \mathrm{p}^{4}\left({ }^{1} \mathrm{D}_{2}\right)\end{array}$ & $\begin{array}{l}379.31 \\
489.50(\mathrm{~g}) \\
1814.63(\mathrm{f}) \\
3869.85(\mathrm{~g}, \mathrm{f})\end{array}$ \\
\hline
\end{tabular}

Notes. The rest-frame wavelengths $\left(\lambda_{0}\right.$ in $\AA$ ) are taken from the CHIANTI atomic database. Ground transitions are labelled with (g). Forbidden transitions are labelled with (f).

The R-matrix ICFT method was used for the scattering calculation of L10 (228 levels) and our work (630 levels). Figure B.2 shows the hexbin plot comparison of the effective collision strengths at three temperatures in the range of $10^{5-7} \mathrm{~K}$. Figure 5 shows the comparison of the effective collision strengths of selected allowed and forbidden transitions from the ground and metastable levels listed in Table 3. The Rmatrix calculations (L10 and our work) agree well for all the highlighted transitions. For the forbidden transitions, the DW collision strengths (Landi \& Bhatia 2006) as available in the CHIANTI atomic database v10.0.1 (Del Zanna et al. 2021a) are systematically lower. 

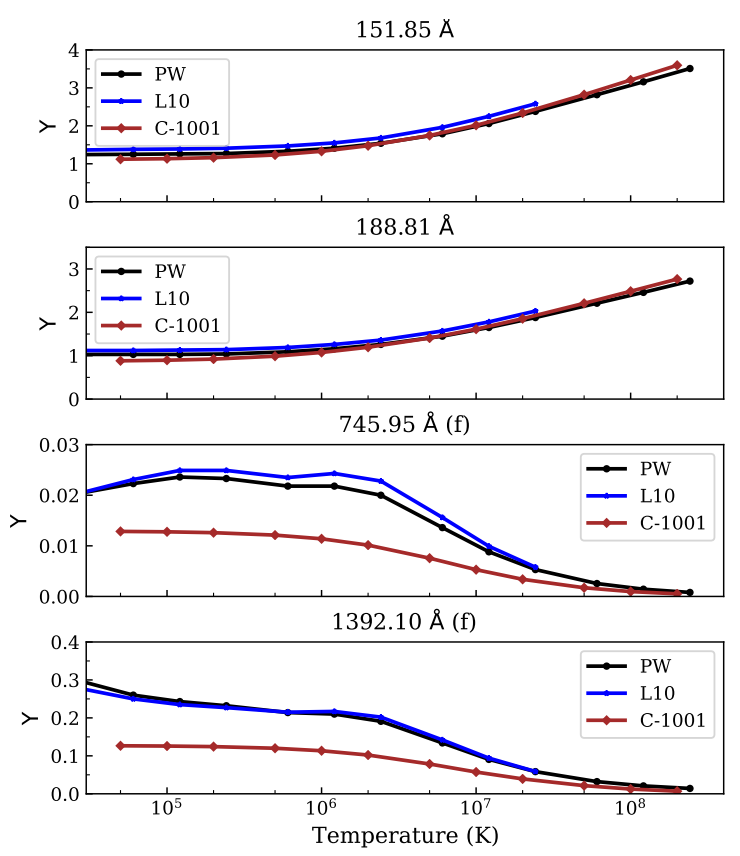

Fig. 5. Comparison of $\mathrm{ArXI}$ (or $\mathrm{Ar}^{10+}$ ) effective collision strengths between our work (M20), Ludlow et al. (2010, L10) and Landi \& Bhatia (2006, DW) as incorporated in the CHIANTI atomic database v10.0.1 (C-1001). The top two panels are allowed transitions from the ground $(188.81 \AA)$ and metastable $(151.85 \AA)$ levels. The bottom two panels are forbidden transitions (745.95 $\AA$ and $1392.10 \AA$ ).

The resonant dipole-allowed transition at $188.81 \AA$ was observed by the Hinode/EIS spectrometer, while the forbidden transitions listed in Table 3, as well as several allowed transitions from the $2 s^{2} 2 p^{3} 3 p$ to $2 s^{2} 2 p^{3} 3 s$ states, have been observed by the SOHO/SUMER instrument in the 715-740 $\AA$ range. These latter transitions, together with the forbidden transition at $745.80 \AA$, are observable by the latest solar UV spectrometer, SPICE (Spice Consortium 2020), on board the ESA mission Solar Orbiter, launched in 2020. These transitions are particularly useful because they are very close in wavelength and could be used to measure the electron temperature, as we show here for the first time.

We considered a SUMER off-limb coronal observation above an active region reported by Curdt et al. (2004) and an Hinode/EIS measurement of the resonant transition (noting that the SUMER and EIS observations were not simultaneous). Figure 6 (top) shows the emissivity ratios of these lines using our atomic data. The emissivity ratios are essentially the ratios of the observed radiances in the lines with their emissivities as a function of the electron temperature for a fixed density, scaled by an arbitrary constant to make the ratios close to unity (see Del Zanna et al. 2004; Del Zanna \& Mason 2018, for details and examples). If a temperature exists for which the line radiances agree with theory, the emissivity ratios would show a crossing.

The emissivities of the chosen lines have a minor dependence on the electron density. We assumed a typical value for an active region, $10^{9} \mathrm{~cm}^{-3}$. To within $20 \%$, predicted and observed emissivities for a typical temperature of an active region $\sim 3 \times 10^{6} \mathrm{~K}$ agree excellently. The only exception is the $736.42 \AA$ line, which is about six times stronger than predicted. This line was listed as the strongest Ar XI line in Curdt et al. (2004), but our atomic data clearly indicate that the $736.42 \AA$ line must be mostly due to another transition. The line is not due to Ar XI. The $20 \%$ scatter
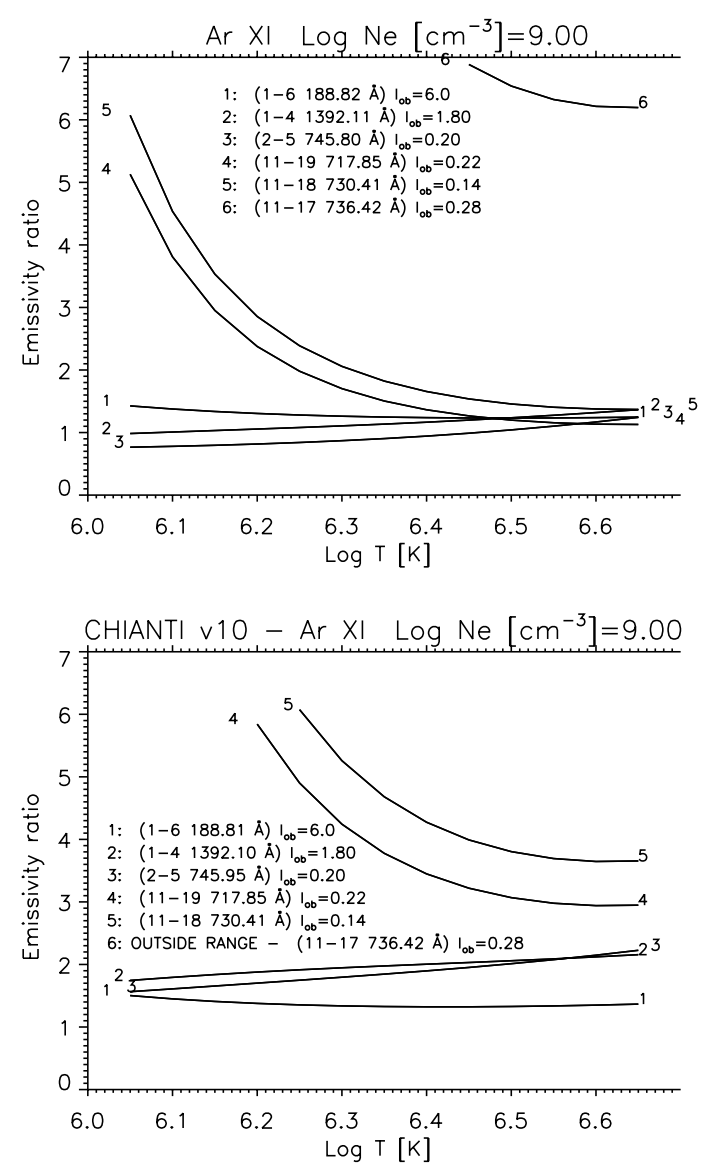

Fig. 6. Emissivity ratios of Ar XI transitions, calculated with our atomic rates (top) and CHIANTI atomic data (bottom). The values in brackets indicate the lower and upper level number and the wavelength $(\AA) . I_{\mathrm{ob}}$ indicates the observed radiances (photon units) in active regions.

could be due to uncertainties in the measurements or calibration, and could be further improved with more accurate radiative data.

Figure 6 (bottom) shows instead the emissivity ratios of the same lines using the CHIANTI atomic data. The emissivity ratio of the resonance line at $188.81 \AA$ is nearly the same, while the ratios of the forbidden lines are significantly higher, reflecting the fact that the emissivities of the lines are much lower, mainly due to the lower DW excitation rates, as shown in Fig. 5. The emissivities of the $3 s-3 p$ transitions $(11-17,11-18,11-19)$ are underestimated by large factors of higher than three.

\subsection{SIX}

The most recent R-matrix calculations of electron-impact excitation data for S IX (or $\mathrm{S}^{8+}$ ) were presented in Liang et al. (2011, L11 hereafter). Like L11, we used AUTOSTRUCTURE to calculate the atomic structure. The top right panels of Figs. 1 and 2 show that the energy levels and transition strengths agree well between our work and L11.

The R-matrix ICFT method was used for the scattering calculation of L11 (92 levels) and our work (630 levels). Figure B.3 shows the hexbin plot comparison of the effective collision strengths at three temperatures in the range of $10^{4-8} \mathrm{~K}$. As shown in Fig. 7, the effective collision strengths of three selected dipole transitions from the ground and metastable levels agree well between the four data sets: our work (PW), Liang et al. (2011, L11), and Bhatia \& Landi (2003, DW) as incorporated in the CHIANTI atomic database v10.0.1 (C-1001). All 


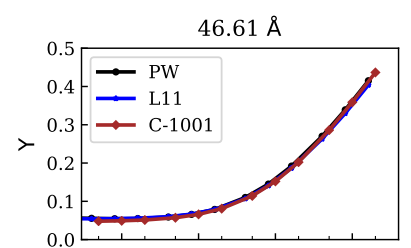

$55.54 \AA$

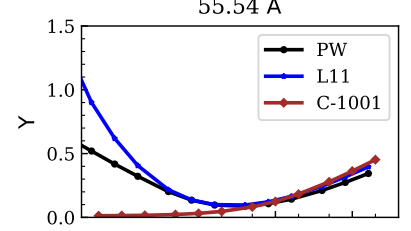

$179.28 \AA$

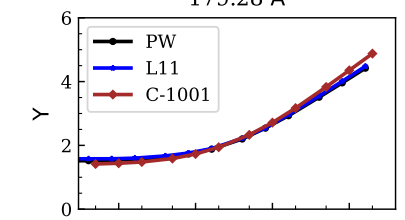

$871.73 \AA$ (f)

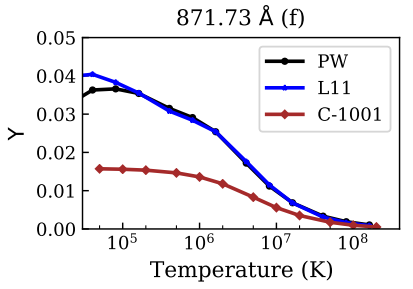

$47.25 \AA$

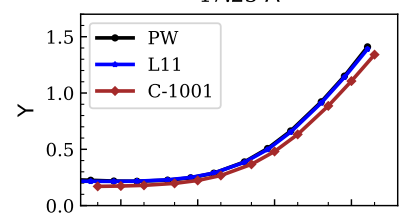

$56.33 \AA$

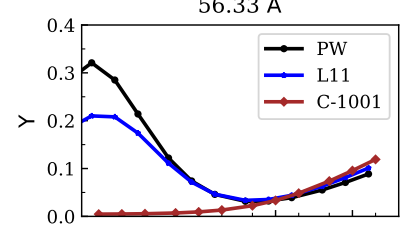

$224.73 \AA$

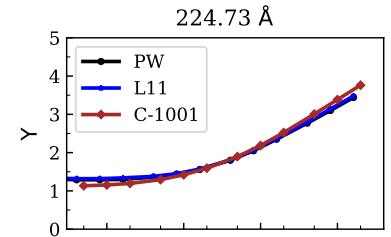

$1715.41 \AA$ (f)

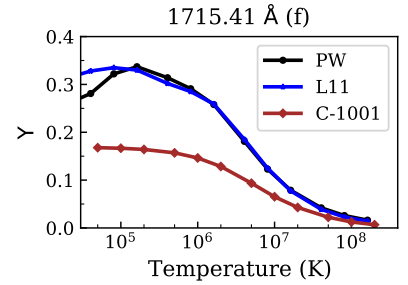

Fig. 7. Comparison of S IX (or $\mathrm{S}^{8+}$ ) effective collision strengths between our work (PW), Liang et al. (2011, L11), and Bhatia \& Landi (2003, DW) as incorporated in the CHIANTI atomic database v10.0.1 (C-1001) for the selected transitions listed in Table 3.

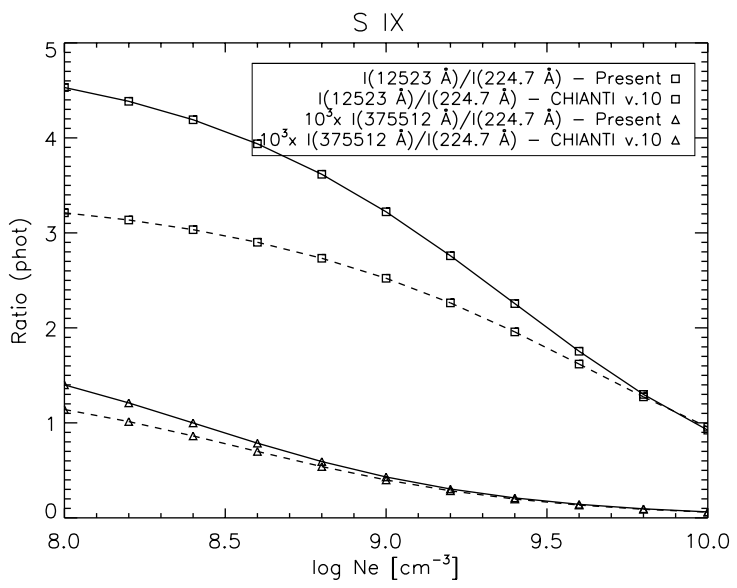

Fig. 8. Ratios of the two main S IX near-infrared transitions, calculated with our atomic rates (full lines) and CHIANTI atomic data (dashed lines), relative to the resonance line at $224.7 \AA$.

three calculations agree well for the transitions of $46.61 \AA$ (metastable), 47.25 $\AA$ (ground), 179.28 $\AA$ (metastable), and $224.73 \AA$ (ground). For the two transitions $55.54 \AA$ and $56.33 \AA$, the three calculations differ at $T \lesssim 10^{6} \mathrm{~K}$. For the two forbidden transitions $871.73 \AA$ and $1715.41 \AA$, the R-matrix data only differ at $T \lesssim 10^{5} \mathrm{~K}$, while the DW data are systematically smaller by a factor of $\lesssim 2$.

Within the ground configuration $\left(2 \mathrm{~s}^{2} 2 \mathrm{p}^{4}\right)$, the main transition is the ${ }^{3} \mathrm{P}_{2}-{ }^{3} \mathrm{P}_{1}$ in the near-infrared (NIR) at $1.25 \mu \mathrm{m}$. It is one of the primary lines for the first large-scale $(4 \mathrm{~m})$ solar ground-based telescope, the Daniel K. Inouye Solar Telescope (DKIST, see Rimmele et al. 2015) and its main NIR instrument, the CryoNIRSP spectropolarimeter (Fehlmann et al. 2016) to measure the sulphur abundance, as this is one of the very few NIR lines to have been observed previously (for a discussion of the diagnostics lines in the NIR, see Del Zanna \& DeLuca 2018). The weaker ${ }^{3} \mathrm{P}_{1}-{ }^{3} \mathrm{P}_{0}$ transition at $3.75 \mu \mathrm{m}$ is also observable by DKIST. Figure 8 shows that with our atomic data, the intensity of the $1.25 \mu \mathrm{m}$ transition is up to $40 \%$ higher than what is calculated by CHIANTI with the DW collisional rates. The forbidden lines in the off-limb observations can be strongly affected by photoexcitation (PE) from the disk radiation, depending on the distance from the photosphere and the local electron density. PE is not included in Fig. 8, but it is included in Fig. 9, where we plot the emissivity ratios of several UV transitions relative to the SUMER off-limb quiet-Sun coronal observation reported by Curdt et al. (2004). We added a measurement from Hinode/EIS of the EUV line at $179.28 \AA$.

As in the Ar XI case, the transitions shown in Fig. 9 provide a very good temperature diagnostic because they are close in wavelength. With our atomic data (top plot), despite the relatively large scatter (about 20\%, mostly in the weaker lines), the curves indicate an electron temperature of $1.4 \times 10^{6} \mathrm{~K}$, in excellent agreement with a few other quiet-Sun measurements, as discussed in Del Zanna \& Mason (2018). On the other hand, using the CHIANTI atomic data, a large scatter (factor of two) in the curves is present, as is shown in Fig. 9 (bottom), and an incorrect temperature would have been estimated.

Finally, we note that a few of the weaker S IX transitions around $715 \AA$ are also observable by the Solar Orbiter SPICE spectrometer. But several identifications reported by Curdt et al. (2004) need to be revised in light of the present atomic data.

\subsection{Si VII}

The most recent R-matrix calculations of electron-impact excitation data for Si VII (or $\mathrm{Si}^{6+}$ ) were presented in Sossah \& Tayal (2014, S14). S14 used the multi-configuration HartreeFock method, which is different from the method we used. The bottom left panel of Fig. 1 shows that the level energies of NIST and S14 agree well, while the energy levels of our work differ by up to $\sim 6 \%$. The transition strengths of NIST, S14, and our work agree well with each other (the bottom left panel of Fig. 2).

S14 used the B-spline R-matrix method for the scattering calculation, including 92 fine-structure target levels. Figure B.4 shows the hexbin plot comparison of the effective collision strengths at three temperatures in the range of $10^{4-6} \mathrm{~K}$. In Fig. 10, we compare the effective collision strengths of selected transitions listed in Table 3. We also considered the Bhatia \& Landi (2003, DW) data as available in CHIANTI. Similar to Ar XI, the three calculations agree well for the allowed transitions, but for the forbidden transitions, the DW rates are systematically lower than those of the R-matrix calculations.

Our rates result in a significant increase in the predicted intensity of the $1049.15 \AA$ transition compared to what is calculated within CHIANTI. We note that the $1049.15 \AA$ transition is one of the primary lines for the Solar Orbiter SPICE instrument to measure chemical abundance variations. Most lines observable by SPICE are due to elements with a high first-ionization potential (FIP) such as $\mathrm{Ne}, \mathrm{Ar}, \mathrm{O}$, and very few transitions from low-FIP elements such as Si are available. These measurements are important as the chemical abundances of low-FIP versus 

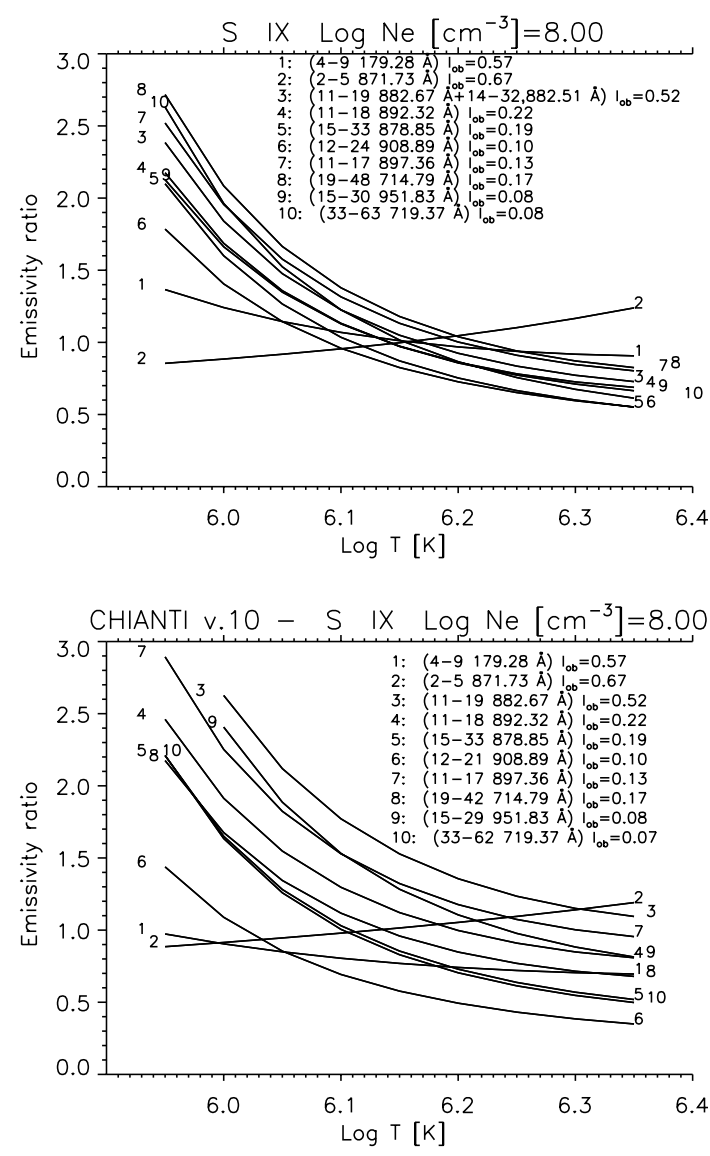

Fig. 9. Emissivity ratios of S IX transitions, calculated with our atomic rates (top) and CHIANTI atomic data (bottom). The values in brackets indicate the lower and upper level number and the wavelength $(\AA) . I_{\mathrm{ob}}$ indicates the observed radiances (photon units) in the quiet Sun.

high-FIP elements in the solar corona and solar wind vary (see the review in Del Zanna \& Mason 2018).

\section{6. $M g V$}

The most recent R-matrix calculations of electron-impact excitation data for $\mathrm{Mg} \mathrm{V}$ ( or $\mathrm{Mg}^{4+}$ ) were presented in Tayal \& Sossah (2015, T15) and Wang et al. (2017, W17). Both T15 and W17 used the multi-configuration Hartree-Fock method. The bottom middle panel of Fig. 1 shows that the level energies of NIST, T15, and W17 agree well among each other, while the energy levels of our work differ by up to $\sim 9 \%$. The transition strengths of NIST, T15, W17, and our work agree well with each other (see the bottom left panel of Fig. 2).

Both T15 and W17 used the B-spline R-matrix method for the scattering calculation. T15, W17, and our work included 86, 316, and 630 fine-structure target levels, respectively. Fig. B.5 shows the hexbin plot comparison of the effective collision strengths at three temperatures in the range of $10^{4-6} \mathrm{~K}$. In Fig. 11 we compare the effective collision strengths of selected transitions listed in Table 3. In general, the data sets agree well.

\subsection{Ne III}

The most recent R-matrix calculations of electron-impact excitation data for Ne III (or $\mathrm{Ne}^{2+}$ ) were presented in McLaughlin et al. (2011, M11 hereafter). Like M11, we used AUTOSTRUCTURE to calculate the atomic structure. The bottom right panel

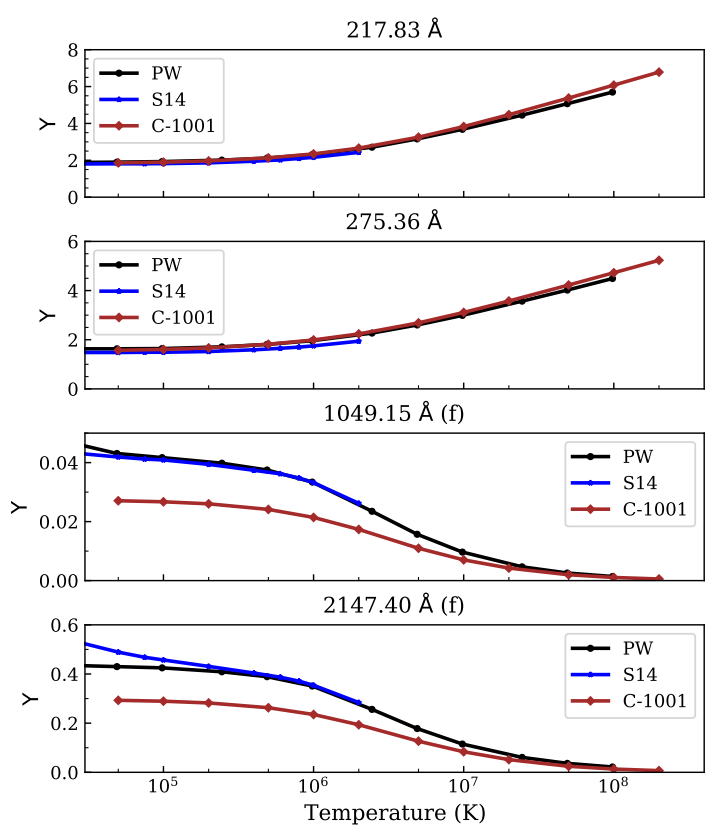

Fig. 10. Comparison of Si VII (or $\mathrm{Si}^{6+}$ ) effective collision strengths between our work (PW), Sossah \& Tayal (2014, S14), and Bhatia \& Landi (2003, DW) as incorporated in the CHIANTI atomic database v10.0.1 (C-1001) for the selected transitions listed in Table 3.

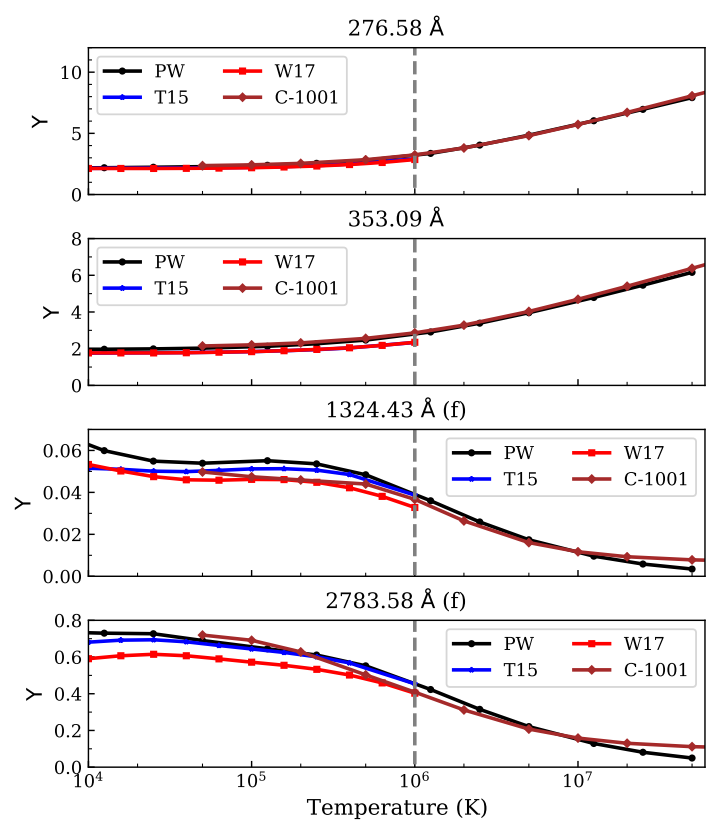

Fig. 11. Comparison of $\mathrm{Mg} \mathrm{V}$ (or $\mathrm{Mg}^{4+}$ ) effective collision strengths between our work (PW), Tayal \& Sossah (2015, T15), Wang et al. (2017, W17), and composite data as incorporated in the CHIANTI atomic database v10.0.1 (C-1001) for the selected transitions listed in Table 3. The vertical dashed lines indicate the temperature threshold $\left(T \sim 10^{5} \mathrm{~K}\right)$ below which R-matrix data from Butler \& Zeippen (1994) were used in CHIANTI, while at higher temperatures, DW data from Bhatia et al. (2006) were used.

of Fig. 1 shows that the level energies of M11 and our work agree well with each other. Both are less accurate $(\lesssim 10 \%)$ than NIST. The transition strengths of NIST, M11, and our work agree well with each other (see the bottom right panel of Fig. 2).

The R-matrix ICFT method was used for the scattering calculation of M11 (554 levels) and our work (630 levels). Figure B.6 

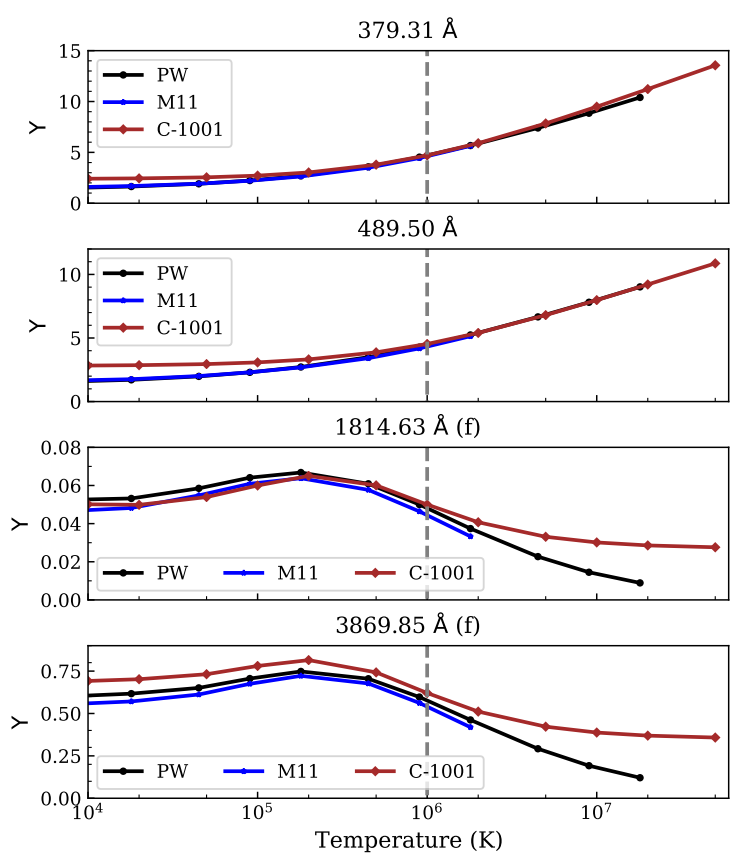

Fig. 12. Comparison of $\mathrm{Ne}$ III (or $\mathrm{Ne}^{2+}$ ) effective collision strengths between our work (PW), McLaughlin et al. (2011, M11), and composite data as incorporated in the CHIANTI atomic database v10.0.1 (C-1001) for the selected transitions listed in Table 3 . The vertical dashed lines indicate the temperature threshold $\left(T \sim 10^{6} \mathrm{~K}\right)$ below which R-matrix data from McLaughlin \& Bell (2000) were used in CHIANTI for transitions within the ground configuration. For other transitions, the DW data from Landi \& Bhatia (2005) were used by CHIANTI.

shows the hexbin plot comparison of the effective collision strengths at three temperatures in the range of $10^{3-6} \mathrm{~K}$.

In Fig. 12, we compare the effective collision strengths of the selected transitions listed in Table 3. Good agreement is found for the allowed transitions, but for the forbidden transitions, the CHIANTI data at $T \gtrsim 10^{6} \mathrm{~K}$ are systematically larger than the R-matrix calculations.

\section{Summary}

We have presented systematic R-matrix intermediate-coupling frame transformation calculations of electron-impact excitation data of O-like ions from Ne III to $\mathrm{Zn}$ XXIII (i.e. $\mathrm{Ne}^{2+}$ to $\mathrm{Zn}^{22+}$ ). For each ion, 630 levels were included in the target configuration interaction and close-coupling collision expansion, which is significantly larger than previous calculations. Level-resolved effective collision strengths were obtained among these levels over a wide temperature range. Because previous R-matrix data were available for only some ions, our work is a significant extension and improvement of electron-impact excitation data of O-like ions. When compared with existing R-matrix data in the atomic databases and literature, generally speaking, the new data provided here are consistent within 0.2 dex at temperatures relevant to astrophysical modelling, which is reassuring. When compared to CHIANTI models that only used DW data (e.g., Ca XIII, Ar XI, S IX, and Si VII), the new data calculated here significantly increase the predicted intensities of many key transitions and improve or provide new plasma diagnostics that are relevant for current high-resolution spectrometers such as the ground-based DKIST/CryoNIRSP, and the space-based Solar Orbiter/SPICE.
Acknowledgements. We thank the referee for careful reading of the manuscript and useful suggestions. The present work is funded by STFC (UK) through the University of Strathclyde UK APAP network grant ST/R000743/1 and the University of Cambridge DAMTP atomic astrophysics group grant ST/T000481/1. J.M. thank A. Giunta and R. Dufresne for useful discussion.

\section{References}

Amato, R., Grinberg, V., Hell, N., et al. 2021, A\&A, 648, A105

Badnell, N. R. 2011, Comput. Phys. Commun., 182, 1528

Badnell, N. R., \& Griffin, D. C. 2001, J. Phys. B At. Mol. Phys., 34, 681

Badnell, N. R., Del Zanna, G., Fernández-Menchero, L., et al. 2016, J. Phys. B At. Mol. Phys., 49, 094001

Barret, D., Lam Trong, T., den Herder, J.-W., et al. 2018, Proc. SPIE, 10699 , $106991 \mathrm{G}$

Berrington, K. A., Eissner, W. B., \& Norrington, P. H. 1995, Comput. Phys. Commun., 92, 290

Bhatia, A. K., \& Landi, E. 2003, At. Data Nuclear Data Tables, 85, 169

Bhatia, A. K., \& Landi, E. 2003, ApJ, 585, 587

Bhatia, A. K., Landi, E., \& Eissner, W. 2006, At. Data Nuclear Data Tables, 92, 105

Butler, K., \& Zeippen, C. J. 1994, A\&AS, 108, 1

Butler, K., \& Badnell, N. R. 2008, A\&A, 489, 1369

Burgess, A. 1974, J. Phys. B At. Mol. Phys., 7, L364

Burgess, A., \& Tully, J. A. 1992, A\&A, 254, 436

Carr, D. B., Littlefield, R. J., Nicholson, W. L., et al. 1987, JASA, 82, 424

Cui, W., Chen, L.-B., Gao, B., et al. 2020, J. Low Temp. Phys., 199, 502

Curdt, W., Landi, E., \& Feldman, U. 2004, A\&A, 427, 1045

Del Zanna, G., \& Mason, H. E. 2018, Liv. Rev. Sol. Phys., 15, 5

Del Zanna, G., \& DeLuca, E. E. 2018, ApJ, 852, 52

Del Zanna, G., Berrington, K. A., \& Mason, H. E. 2004, A\&A, 422, 731

Del Zanna, G., Badnell, N. R., Fernández-Menchero, L., et al. 2015, MNRAS, 454, 2909

Del Zanna, G., Dere, K. P., Young, P. R., et al. 2021a, ApJ, 909, 38

Del Zanna, G., Andretta, V., Cargill, P. J., et al. 2021b, Front. Astron. Space Sci., 8,33

Dere, K. P., Landi, E., Mason, H. E., et al. 1997, A\&AS, 125, 149

Eissner, W., Jones, M., \& Nussbaumer, H. 1974, Comput. Phys. Commun., 8, 270

Fehlmann, A., Giebink, C., Kuhn, J. R., et al. 2016, Proc. SPIE, 9908, 99084D.

Feldman, U., Doschek, G. A., Nagel, D. J., et al. 1973, ApJ, 183, L43

Fernández-Menchero, L., Giunta, A. S., Del Zanna, G., et al. 2016, J. Phys. B At. Mol. Phys., 49, 085203

Grinberg, V., Hell, N., El Mellah, I., et al. 2017, A\&A, 608, A143

Junjie M. 2021, http: //doi . org/10.5281/zenodo. 5103521

Kaastra, J. S., Mewe, R., \& Nieuwenhuijzen, H. 1996, UV and X-ray Spectroscopy of Astrophysical and Laboratory Plasmas, 411

Kaastra, J. S., Raassen, A. J. J., de Plaa, J., \& Gu, Liyi. 2020, http: //doi . org/ 10.5281/zenodo. 4384188

Landi, E., \& Bhatia, A. K. 2005, A\&A, 444, 305

Landi, E., \& Bhatia, A. K. 2005, At. Data Nuclear Data Tables, 89, 195 Landi, E., \& Bhatia, A. K. 2006, At. Data Nuclear Data Tables, 92, 305 Li, F., Liang, G. Y., \& Zhao, G. 2013, ApJ, 762, 53

Liang, G. Y., Badnell, N. R., Zhao, G., et al. 2011, A\&A, 533, A87

Ludlow, J. A., Ballance, C. P., Loch, S. D., et al. 2010, J. Phys. B At. Mol. Phys., 43, 074029

Mao, J., Kaastra, J. S., Mehdipour, M., et al. 2017, A\&A, 607, A100

Mao, J., Mernier, F., Kaastra, J. S., et al. 2019, J. Instrum., 14, C07012

Mao, J., Badnell, N. R., \& Del Zanna, G. 2020, A\&A, 634, A7

Mao, J., Badnell, N. R., \& Del Zanna, G. 2020, A\&A, 643, A95

McLaughlin, B. M., \& Bell, K. L. 2000, J. Phys. B At. Mol. Phys., 33, 597

McLaughlin, B. M., Lee, T.-G., Ludlow, J. A., et al. 2011, J. Phys. B At. Mol Phys., 44, 175206

Nussbaumer, H., \& Storey, P. J. 1978, A\&A, 64, 139

Phillips, K. J. H., Fawcett, B. C., Kent, B. J., et al. 1982, ApJ, 256, 774

Raju, P. K., \& Dwivedi, B. N. 1978, Sol. Phys., 60, 269

Rimmele, T., McMullin, J., Warner, M., et al. 2015, IAU General Assembly, \#29, id. 2255176

Sossah, A. M., \& Tayal, S. S. 2014, ApJ, 787, 2

Smith, R. K., Abraham, M. H., Allured, R., et al. 2016, Proc. SPIE, 99054M

Spice Consortium (Anderson, M., et al.) 2020, A\&A, 642, A14

Tayal, S. S., \& Sossah, A. M. 2015, A\&A, 574, A87

Wang, T. J., Innes, D. E., \& Solanki, S. K. 2006, A\&A, 455, 1105

Wang, K., Jönsson, P., Ekman, J., et al. 2017, ApJS, 229, 37 


\section{Appendix A: Scaling parameters}

We list the Thomas-Fermi-Dirac-Amaldi potential scaling parameters used in the AUTOSTRUCTURE calculations in Table A.1. These scaling parameters were obtained in the same way for all the O-like ions considered here without further manual adjustment.

\section{Appendix B: Hexbin plot comparisons}

A large number of effective collision strengths were calculated by our and previous $R$-matrix calculations for some O-like ions. Following Mao et al. (2020a) and Mao et al. (2020b), hexbin plots were used (Carr et al. 1987) to compare these results.

The hexbin plot of Ne III behaves slightly differently than the other ions shown here, exhibiting increased scatter with an increase temperature from $T=9.00 \times 10^{4} \mathrm{~K}$ to $T=1.80 \times 10^{6} \mathrm{~K}$. The scatter is still relatively large (several orders of magnitude) for some transitions. We should point out that the percentage of transitions that differ by at least 0.2 dex $(\sim 25 \%)$ is still comparable to that of Fe XIX (Table 2). When we focus on transitions from the lowest five energy levels alone, good agreement is found with our work (Table 2, see also Fig. 12).

According to McLaughlin et al. (2011, M11), 20 continuum basis orbitals were used by M11 during their calculation to keep the dimensions of the Hamiltonian matrix at a more manageable size. This is because their primary focus was line ratios between transitions within the ground configuration. That is to say, the transitions among high-lying levels are less accurate for M11.

In our work, 40 continuum basis orbitals were used for Ne III. The maximum basis orbital energy (ranging from 14.83 Ryd for $L=4$ to 20.76 Ryd for $L=19$ ) we covered is a factor of $\sim 3.2-4.5$ higher than the ionization potential (4.66 Ryd) of Ne III. We performed an atomic structure calculation using the 24 configurations and the ten scaling parameters specified in M11. Subsequently, we performed an inner-region exchange calculation with 20 continuum basis orbitals and included angular momenta up to $2 J=23$ (i.e. up to $L=19$ ). In this exercise, the $R$-matrix radius was 22.88 a.u. (as in M11) and the maximum basis orbital energy ranged from 8.04 Ryd for $L=4$ to 14.72 Ryd for $L=19$, which is a factor of $1.7-3.2$ the ionization potential (4.66 Ryd) of Ne III. 

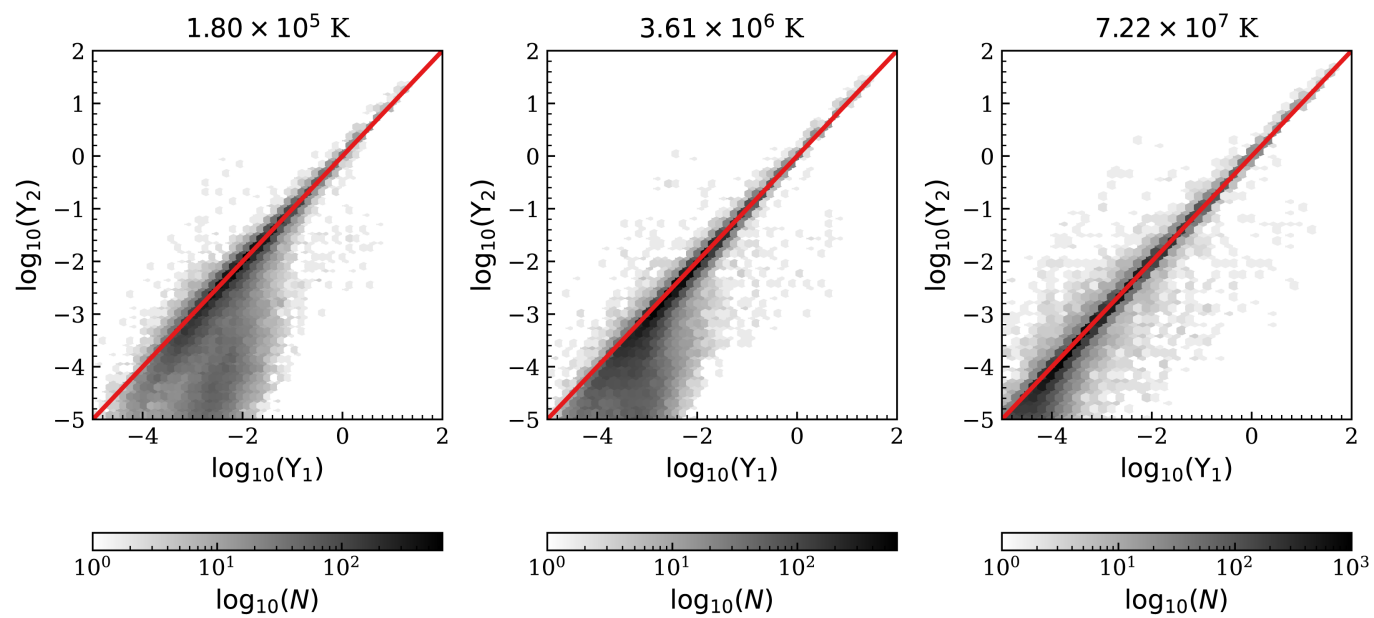

Fig. B.1. Hexbin plots of the comparison of the Fe XIX (or Fe ${ }^{18+}$ ) effective collision strengths between our work $\left(\Upsilon_{1}\right)$ and Butler \& Badnell $(2008$, $\Upsilon_{2}$ ) at $T=1.80 \times 10^{5} \mathrm{~K}$ (left) and $3.61 \times 10^{6} \mathrm{~K}$ (middle), and $7.22 \times 10^{7} \mathrm{~K}$ (right). The darker the colour, the greater the number of transitions $\log _{10}(N)$. The diagonal line in red indicates $\Upsilon_{1}=\Upsilon_{2}$.
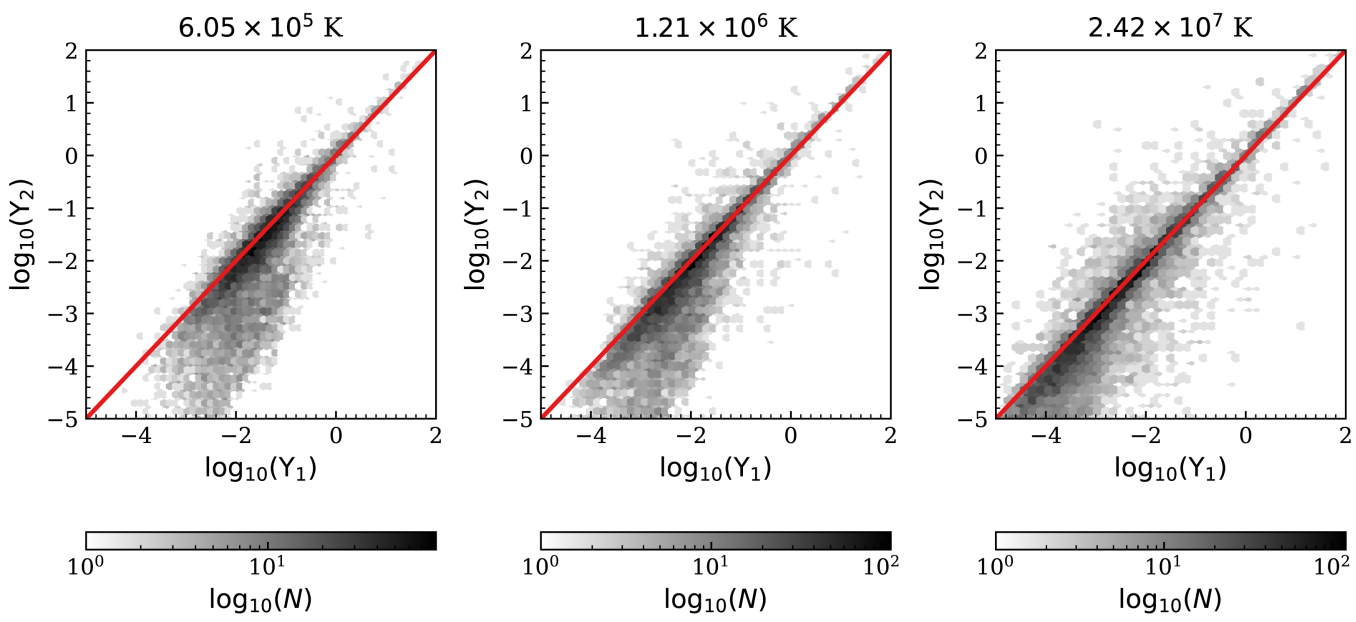

Fig. B.2. Hexbin plots of the comparison of the Ar XI (or $\left.\mathrm{Ar}^{10+}\right)$ effective collision strengths between our work $\left(\Upsilon_{1}\right)$ and Ludlow et al. $\left(2010, \Upsilon_{2}\right)$ at $T=6.06 \times 10^{5} \mathrm{~K}$ (left) and $1.21 \times 10^{6} \mathrm{~K}$ (middle), and $2.42 \times 10^{7} \mathrm{~K}$ (right). The darker the colour, the greater the number of transitions $\log _{10}(N)$. The diagonal line in red indicates $\Upsilon_{1}=\Upsilon_{2}$.
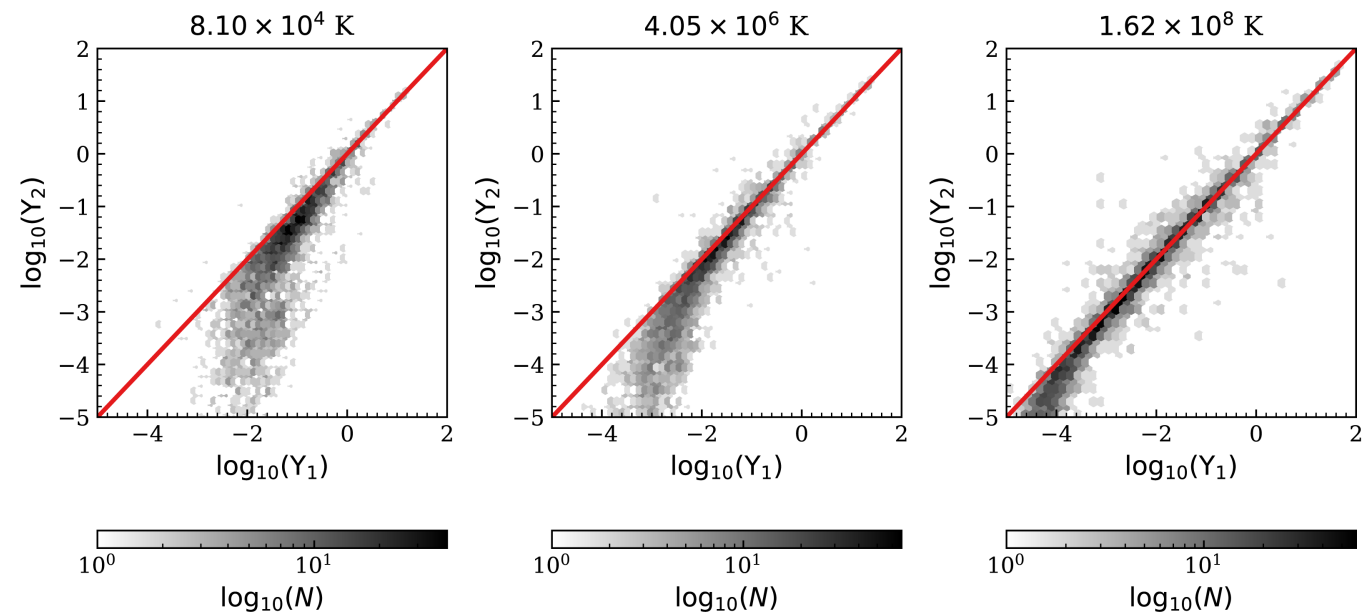

Fig. B.3. Hexbin plots of the comparison of the S IX (or $\mathrm{S}^{8+}$ ) effective collision strengths between our work $\left(\Upsilon_{1}\right)$ and Liang et al. $\left(2011\right.$, $\left.\Upsilon_{2}\right)$ at $T=8.10 \times 10^{4} \mathrm{~K}$ (left) and $4.05 \times 10^{6} \mathrm{~K}$ (middle), and $\sim 1.62 \times 10^{8} \mathrm{~K}$ (right). The darker the colour, the greater the number of transitions $\log _{10}(N)$. The diagonal line in red indicates $\Upsilon_{1}=\Upsilon_{2}$. 

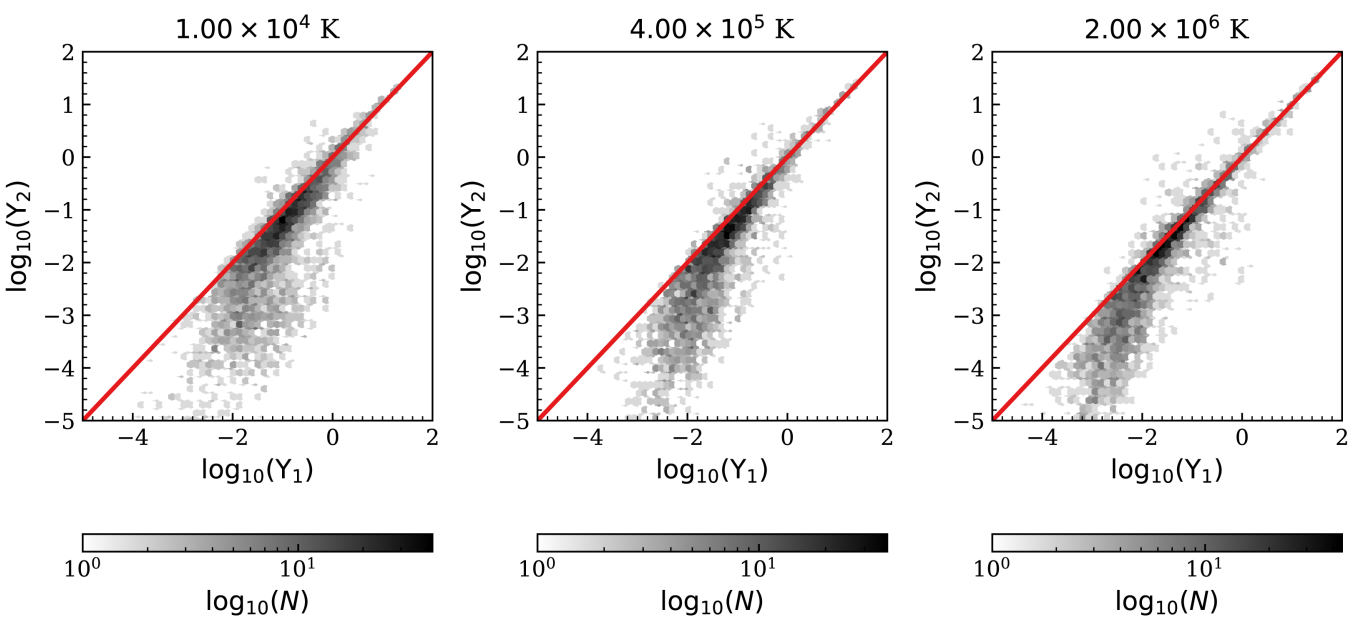

Fig. B.4. Hexbin plots of the comparison of the Si VII $\left(\right.$ or $\mathrm{Si}^{6+}$ ) effective collision strengths between our work $\left(\Upsilon_{1}\right)$ and Sossah \& Tayal (2014, $\Upsilon_{2}$ ) at $T=1.00 \times 10^{4} \mathrm{~K}$ (left) and $4.00 \times 10^{5} \mathrm{~K}$ (middle), and $2.00 \times 10^{6} \mathrm{~K}$ (right). The darker the colour, the greater the number of transitions $\log _{10}(N)$. The diagonal line in red indicates $\Upsilon_{1}=\Upsilon_{2}$.
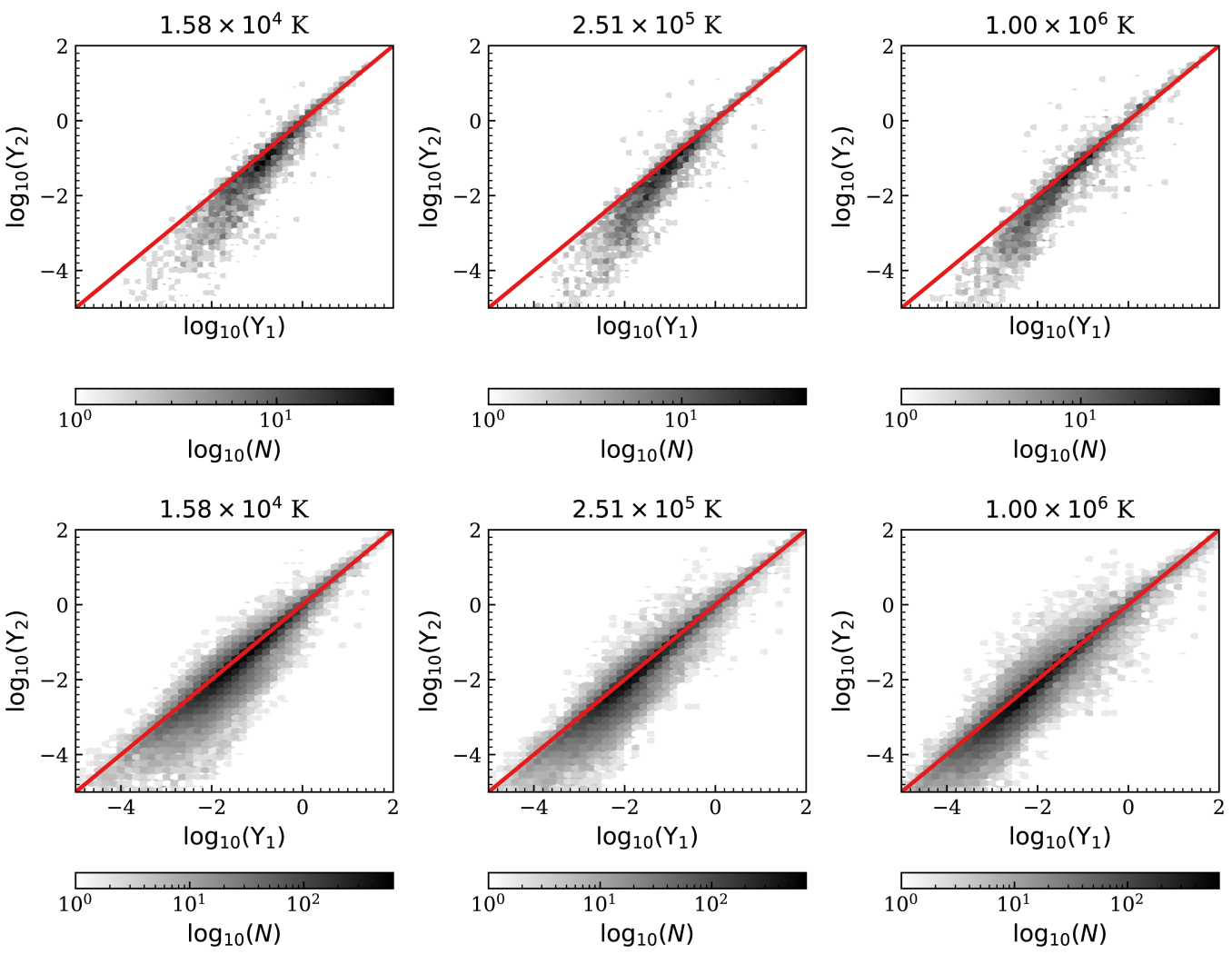

Fig. B.5. Hexbin plots of the comparison of the $\mathrm{Mg} \mathrm{V}$ (or $\mathrm{Mg}^{4+}$ ) effective collision strengths between our work ( $\left.\Upsilon_{1}\right)$ and Tayal \& Sossah (2015, $\Upsilon_{2}$ ) (upper) or Wang et al. (2017, $\Upsilon_{2}$ ) (bottom) at $T=1.58 \times 10^{4} \mathrm{~K}$ (left) and $2.51 \times 10^{5} \mathrm{~K}$ (middle), and $1.00 \times 10^{6} \mathrm{~K}$ (right). The darker the colour, the greater the number of transitions $\log _{10}(N)$. The diagonal line in red indicates $\Upsilon_{1}=\Upsilon_{2}$. 

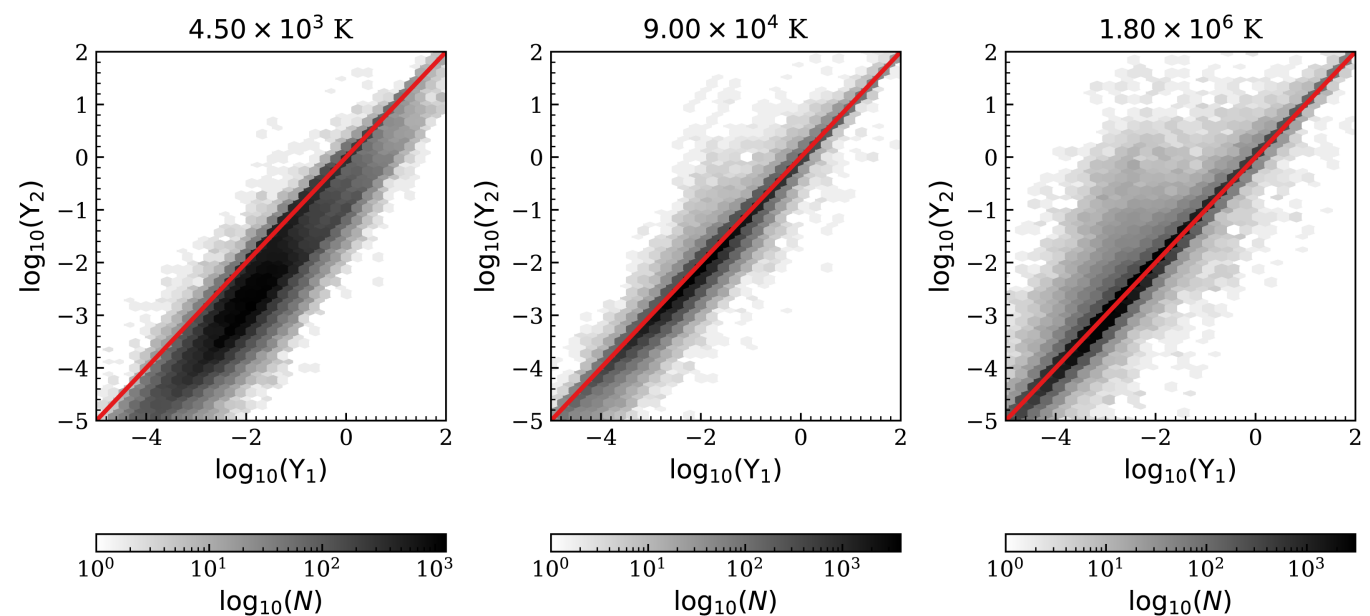

Fig. B.6. Hexbin plots of the comparison of the Ne III (or Ne ${ }^{2+}$ ) effective collision strengths between our work $\left(\Upsilon_{1}\right)$ and McLaughlin et al. $(2011$, $\Upsilon_{2}$ ) at $T=4.50 \times 10^{3} \mathrm{~K}$ (left) and $9.00 \times 10^{4} \mathrm{~K}$ (middle), and $1.80 \times 10^{6} \mathrm{~K}$ (right). The darker the colour, the greater the number of transitions $\log _{10}(N)$. The diagonal line in red indicates $\Upsilon_{1}=\Upsilon_{2}$. 
A\&A 653, A81 (2021)

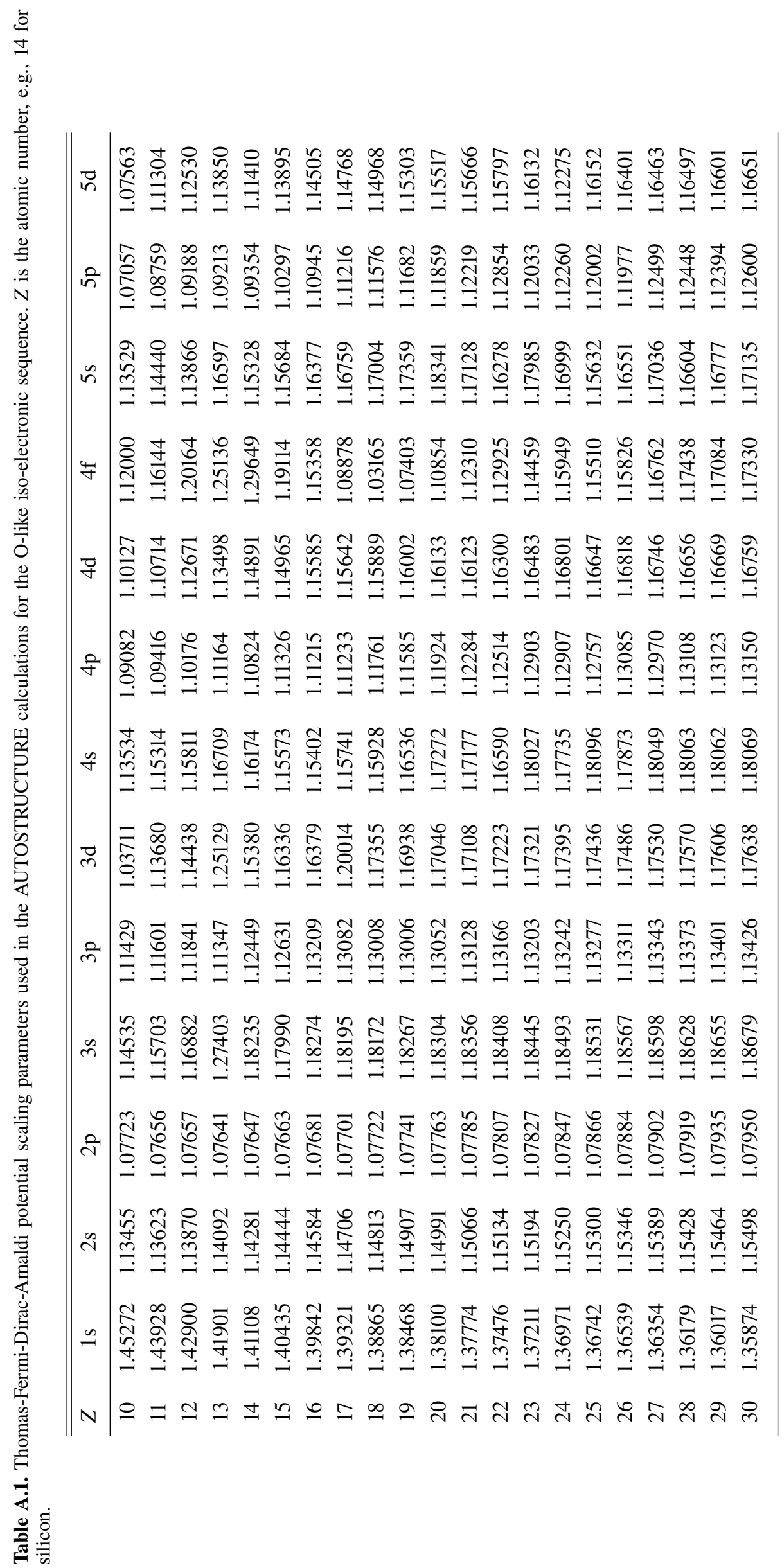

